\title{
PARABOLIC SCHEMES FOR QUASI-LINEAR PARABOLIC AND HYPERBOLIC PDES VIA STOCHASTIC CALCULUS
}

\author{
SEBASTIEN DARSES AND EMMANUEL DENIS
}

\begin{abstract}
We consider two quasi-linear initial-value Cauchy problems on $\mathbb{R}^{d}$ : a parabolic system and an hyperbolic one. They both have a first order non-linearity of the form $\phi(t, x, u) \cdot \nabla u$, a forcing term $h(t, x, u)$ and an initial condition $u_{0} \in L^{\infty}\left(\mathbb{R}^{d}\right) \cap C^{\infty}\left(\mathbb{R}^{d}\right)$, where $\phi$ (resp. $h$ ) is smooth and locally (resp. globally) Lipschitz in $u$ uniformly in $(t, x)$. We prove the existence of a unique global strong solution for the parabolic system. We show the existence of a unique local strong solution for the hyperbolic one and we give a lower bound regarding its blow up time. In both cases, we do not use weak solution theory but recursive parabolic schemes studied via a stochastic approach and a regularity result for sequences of parabolic operators. The result on the hyperbolic problem is performed by means of a non-classical vanishing viscosity method.
\end{abstract}

Key words: Quasi-linear Parabolic PDEs, Hyperbolic PDEs systems, Vanishing viscosity method, Smooth solutions, Stochastic Calculus, Feynman-Kac Formula, Girsanov's Theorem.

\section{INTRODUCTION}

The PDE problems we are studying in the current paper are part of the so-called class of quasi-linear parabolic and hyperbolic initial-value systems:

$$
\left\{\begin{array}{l}
\partial_{t} u+\sum_{j=1}^{d} A_{j}(t, x, u) \partial_{j} u-\sum_{i, j} a_{i j}(t, x) \partial_{i j}^{2} u=h(t, x, u) \\
u(0, x)=u_{0},
\end{array}\right.
$$

where $(t, x) \in \mathbb{R}^{d}, A_{j}$ are $d \times d$ matrices, and $a$ is a positive definite matrix in the parabolic case and $a=0$ in the hyperbolic one. There exists a huge literature regarding analytical methods proving the existence of solution for such PDEs. We refer to [16], [18] and references therein regarding both cases.

We are interested in applying stochastic methods to show existence results when the matrices $A_{j}$ are diagonal. More precisely, we are here concerned with the following case: the first order non-linearity has the special form $\phi(t, x, u) \cdot \nabla u$. The map $\phi:[0, T] \times \mathbb{R}^{d} \times \mathbb{R}^{d} \rightarrow \mathbb{R}^{d}$ (resp. $h$ ) is assumed to be smooth and locally (resp. globally) Lipschitz in $u$ uniformly in $(t, x)$. We prove global and local existence of smooth solutions in $\mathbb{R}^{d}$, respectively in the parabolic case and the hyperbolic one. We handle these questions by means of probabilistic methods due to the relationship between the structure $b \cdot \nabla u$ and the Feynman Kac formula for parabolic equations. More precisely, we use in both cases some iterative parabolic schemes $\left(u_{n}\right)$ where the non linearity 
$\phi(t, x, u) \cdot \nabla u$ is replaced by $\phi\left(t, x, u_{n}\right) \cdot \nabla u_{n+1}$. Let us now describe in more details the two main results of our work.

\subsection{Description of the main results.}

1.1.1. Parabolic case. In Section 3, we prove the following theorem. Let $v_{0} \in L^{\infty}\left(\mathbb{R}^{d}\right) \cap C^{\infty}\left(\mathbb{R}^{d}\right)$ with bounded derivatives. Assume that $h(t, x, v)$ is a globally Lipschitz function in $u$ uniformly in $(t, x)$. Then, for any given time $T>0$, there exists a unique smooth solution $v$ on $[0, T]$ verifying the Cauchy problem

$$
\left\{\begin{array}{l}
\partial_{t} v(t, x)+(\phi(t, x, v) \cdot \nabla) v(t, x)-\frac{1}{2} \sum_{i, j} a_{i j}(t, x) \partial_{i j}^{2} v(t, x)=h(t, x, v(t, x)) \\
v(0, x)=v_{0}(x),
\end{array}\right.
$$

where $a:[0, T] \times \mathbb{R}^{d} \rightarrow \mathbb{R}^{d} \otimes \mathbb{R}^{d}$ satisfies suitable smooth and boundedness conditions on its derivatives. We use the aforementioned iterative scheme $\left(v_{n}\right)$ defined by

$$
\begin{aligned}
\partial_{t} v_{n+1}(t, x)+\left(\phi\left(t, x, v_{n}\right) \cdot \nabla\right) v_{n+1}(t, x)-\frac{1}{2} \Delta_{\sigma} v_{n+1}(t, x) & =h\left(t, x, v_{n}(t, x)\right) \\
v_{0}(t, x) & =v_{0}(x),
\end{aligned}
$$

where $\Delta_{\sigma}$ denotes the second order term.

By means of Girsanov's transformations on the Feynam Kac representation of the solution $v_{n+1}$, we show that $\left(v_{n}\right)$ converges to a vector field $v$ and we obtain uniform bounds for the spatial derivatives of $\left(v_{n}\right)$. We conclude about the regularity of the solution $v$ by verifying the suitable hypothesis of Friedman's theorem on the convergence of parabolic operators [9]. We finally show the uniqueness of the solution. Again, Girsanov's transformations are helpful. We stress that our method does not use the theory of weak solution for PDEs and does not involve fixed point theorems due to the direct study of inductive schemes and some related induction hypothesis. The conditions on $h$ and $\sigma$ we use are sufficient to ensure the existence of a global smooth solution. Let us note that this global existence may fail to hold for $v_{0} \in L^{\infty}$ if the source term is no longer globally Lipschitz in $u$. For instance, consider the one-dimensional case $h(t, x, v)=v^{1+\varepsilon}, \varepsilon>0$ with a constant initial condition $v_{0}(x)=v_{0}>0$. Then the function

$$
v(t, x)=\left(\frac{1}{v_{0}^{-\varepsilon}-\varepsilon t}\right)^{1 / \varepsilon}
$$

is a blow-up solution of (1.2). Our theorem may then be seen as a critical case in that sense. We also show in Section 3.2 the local existence of a smooth solution when $h(t, x, v)=v^{\alpha}$ for general initial conditions.

1.1.2. Hyperbolic case. In Section 4, we prove by means of a particular parabolic scheme a local existence result for a system of quasi linear hyperbolic PDEs when the initial condition $v_{0}$ is a smooth bounded function with bounded derivatives of all orders. We claim that there exists $T^{*}>0$ and a unique smooth solution $v$ on $\left[0, T^{*}\right)$ verifying the Cauchy problem

$$
\left\{\begin{aligned}
\partial_{t} v(t, x)+(\phi(t, x, v) \cdot \nabla) v(t, x) & =h(t, x, v(t, x)) \\
v(0, x) & =v_{0}(x) .
\end{aligned}\right.
$$


Let us describe the proof of this claim. First, we study a collection of sequences $\sigma \mapsto u^{\sigma}:=\left(u_{n}^{\sigma}\right)$ derived from a Feynman-Kac representation where the time-dependent coefficient diffusion sequence $\sigma=\left(\sigma_{n}(t)\right)_{n}$ lies in a family of smooth positive functions. We obtain uniform $L^{\infty}$ bounds in $n$ and $\sigma$ of the derivatives of $\left(u_{n}^{\sigma}\right)$ on a small interval. We show the uniform convergence of a particular sequence $u^{*}:=\left(u_{n}^{\sigma^{*}}\right)$ for a suitable sequence $\sigma^{*}$ converging to 0 as $n \rightarrow \infty$. We conclude that the limit $u^{*}$ of $\left(u_{n}^{\sigma^{*}}\right)$ is a smooth solution of the Cauchy system by adding a constant diffusion term on both side before applying Friedman's Theorem. The proof actually relies on estimates regarding the difference $u_{n+1}-u_{n}$ and on a suitable conjecture on the structure of the sequence $\sigma^{*}$. Here we do not use Girsanov's transformation but rather estimates coming directly from the underlying diffusion process in the Feynman Kac representation. Finally, we provide a lower bound regarding the blow up time for this hyperbolic system.

Section 2 is devoted to some notational conventions. The Appendix recalls the key result of Friedman to obtain in the parabolic case the regularity of the solution through subsequences of parabolic operators.

\subsection{Our contribution related to known litterature.}

\subsubsection{Parabolic case.}

Known results. In the huge literature on parabolic PDEs, one may distinguish the results obtained by purely PDEs tools and those by probabilistic tools:

(i) PDEs point of view. Local existence results are well known for more general semilinear parabolic PDEs (cf Proposition 1.1 p.273 in [18]). One may classify the various studies depending on considering a scalar equation or a system of equations on one hand, and bounded or unbounded domains on the other hand.

In the case $u: \mathbb{R}^{d} \rightarrow \mathbb{R}$, a very general result on the global solvability is given by Theorem 8.1 (Chap. 5 p. 495) in the seminal book [12].

Let us now consider quasi-linear uniformly parabolic systems. First, consider the case of bounded domains. One may mention Theorem 7.1 p.596 in [12, Chap. 7] when the initial condition belongs to some Sobolev spaces. As [12, Th. 8.1], [12, Th. 8.1] may provide existence and unicity of a classical solution under Holder continuity conditions on the coefficients. Those coefficients may also depend on the solution and its gradient. In the case of square integrable initial conditions only, let us refer the reader to Example 3.6 p.75 in [10] where the author also treats general parabolic PDEs. Second, in the case of $\mathbb{R}^{d}$, it can be proven that parabolic PDEs satisfying the following assumptions: "symmetric" first order non-linearity, $h=0, a=c s t$.Id and $v_{0} \in L^{2}\left(\mathbb{R}^{d}\right)$, have a unique global solution (cf Proposition 1.4 p.276 in [18]).

(ii) Probabilistic point of view. First, let us notice that representing parabolic PDEs by means of Feynman-Kac formula is a widely used tool. It even gives results about weak solutions of non-linear PDEs (see e.g. [3]).

The quasi-linear parabolic PDEs we consider can be naturally related to some backward stochastic differential equations. Pardoux and Peng developed a theory of Backward Stochastic Differential Equations and its connection with a wide class of solutions of quasi linear parabolic PDEs, as viscosity solutions for instance (for an overview, see e.g. [17]). In the case we are interested in, that is a non-degenerate system of quasi-linear 
PDEs defined on $\mathbb{R}^{d}$, various results have been obtained in $[14,6]$ for instance. The coefficients may depend on $u$ and $\nabla u$. Under the assumption that the coefficients are smooth and have bounded derivatives in $x, u$ and $\nabla u$ (cf Assumption $\left(A_{1}\right)$ p.343 in [14]) and other specific bounded assumptions (cf Assumption $\left(A_{3}\right)$ and $\left(A_{4}\right)$ p.343 in [14]), the authors prove [14, Prop. 3.3] that the system has a unique smooth and global solution. They prove the corresponding existence result for the related BSDE. Those results are relaxed in [6], cf Assumptions $\left(A_{2}\right)$ [6, p. 229]. The author states one of his main results [6, Th. 2.6 p. 240] on the existence and uniqueness of solutions for the involved BSDE. Finally he proves [6, Corollary. B.7 p. 281] that the related PDEs system has a unique bounded classical solution globally. Both in [6] and [14], the linear growth of all the coefficients in $u$ and $\nabla u$ are basically required.

Roughly speaking, the global solvability results in the PDE literature hold on bounded domains or require integrability conditions on the initial condition when considering $\mathbb{R}^{d}$; The corresponding results on $\mathbb{R}^{d}$ in the stochastic literature hold under linear growth conditions (see e.g. $[14,6]$ ).

Our contribution. This one is twofold:

(1) Concerning the result in its own right, we do not require, in the $\mathbb{R}^{d}$ case, either the advection coefficient to have linear growth in $u$, or integrability conditions on the initial condition to prove the existence of a unique global smooth solution. (As discussed above in 1.1.1, the linear growth in $u$ of the forcing term is a necessary condition to ensure the existence and uniqueness of a global smooth solution.) Though the result might be expected in view of all the above-mentioned discussions, it seems to us that it does not exist in the literature, up to our best knowledge. We overcome the involved difficulties by means of induction hypotheses on the Picard scheme (1.6-1.7).

(2) Regarding the method, we did not find a specific reference that studies such global iterative schemes for these quasi-linear parabolic PDEs. Notice that an interesting scheme has been studied in [2] when a system (written as $(1.2)$ with $h=0$ ) is viewed as a scalar $\mathrm{PDE}$ in an extended phase space.

Let us stress that we identify the rate of the global strong convergence in $C_{0}\left([0, T] \times \mathbb{R}^{d}\right)$ of the linear (and explicit) parabolic PDE sequence (1.6-1.7) to the smooth solution of the non linear system. Surprisingly, it is the same as the one of the standard scheme

$$
X_{t}^{n+1}=x_{0}+\int_{0}^{t} b\left(s, X_{s}^{n}\right) d s+\int_{0}^{t} \sigma\left(s, X_{s}^{n}\right) d W_{s}
$$

converging in $L^{2}\left([0, T] \times \mathbb{R}^{d}\right)$ to the solution of the classical stochastic differential equation

$$
X_{t}=x_{0}+\int_{0}^{t} b\left(s, X_{s}\right) d s+\int_{0}^{t} \sigma\left(s, X_{s}\right) d W_{s},
$$

under linear growth and Lipschitz conditions on the coefficients.

\subsubsection{Hyperbolic case.}

Known results. The literature on Hyperbolic PDEs is also huge and our references could not be by no means complete. Let us refer to [7] and [18] for an overview on basic results.

Iterative hyperbolic schemes of the type

$$
\begin{aligned}
\partial_{t} v_{n+1}(t, x)+\left(\phi\left(t, x, v_{n}\right) \cdot \nabla\right) v_{n+1}(t, x) & =h\left(t, x, v_{n}(t, x)\right) \\
v_{0}(t, x) & =v_{0}(x),
\end{aligned}
$$


are classical to show the local existence of solutions in Sobolev spaces for non linear symmetric hyperbolic PDEs (see e.g. [15]). These results are usually stated and proven using Sobolev estimates, see e.g. [16, Th. 3.2 p.22]. Stochastic methods are not used often to handle these estimates. We may however mention that linear transport equation with Sobolev coefficient has been extended to SDE, see [1].

The study of hyperbolic systems is sometimes performed via vanishing viscosity methods. Such methods have been extensively studied for instance for hyperbolic systems in one-space dimension. Let us refer to the seminal paper [4] regarding various advanced results. A vanishing viscosity method has been also studied in [2] via stochastic methods for a system of Hyperbolic PDEs with no forcing term.

Our contribution. We do not require a priori the initial data to verify integrable conditions on $\mathbb{R}^{d}$. If $v_{0}$ belongs to $L^{2}\left(\mathbb{R}^{d}\right)$ in addition, our result turns out to be a special case of a well known result regarding symmetric hyperbolic systems (cf e.g. [15] Th.2.1 p.30).

We moreover identify a lower bound for the blow-up time that only depends on the data of the Cauchy problem.

We actually perform a non classical vanishing viscosity method for a system of hyperbolic PDEs via a stochastic approach. The vanishing viscosity method we use is non-classical in the sense that the viscosity vanishes in a very specific way through a sequence of linear parabolic PDEs.

\section{Notations}

2.1. General Notations. Fix $T>0$ and let $d$ be a positive integer. The space $\mathbb{R}^{d}$ is endowed with its usual canonical scalar product: if $x, y \in \mathbb{R}^{d}$, we denote by $x \cdot y$ their scalar product. Let $|\cdot|$ be the induced norm. More generally, the notation $|\cdot|$ still denotes the operator norm of any multilinear form. For instance, $|\mathrm{Id}|=1$ where $\mathrm{Id} \in \mathbb{R}^{d} \otimes \mathbb{R}^{d}$.

We denote by $C_{b, k}^{K}\left(\mathbb{R}^{d}\right)$ the space of continuously $K$-times differentiable bounded functions with bounded derivatives of all order $p \leq k(0 \leq k \leq K \leq \infty)$.

We set $\|f(t)\|_{\infty}=\sup _{x \in \mathbb{R}^{d}}|f(t, x)|$ and $\|f\|_{\infty}=\sup _{t \in[0, T]}\|f(t)\|_{\infty}$ when $f:[0, T] \times \mathbb{R}^{d} \rightarrow \mathbb{R}^{d}$ is a continuous function. If $f(t, \cdot) \in L^{p}\left(\mathbb{R}^{d}\right),\|f(t)\|_{p}$ denotes the $L^{p}$ norm of $f(t, \cdot), 1 \leq p<+\infty$.

If $r>0$, we set $B(0, r):=\left\{x \in \mathbb{R}^{d},|x| \leq r\right\}$.

Throughout the paper, $C$ denotes some constant independent of $n$, which can change from line to line. When a particular constant $\mathrm{C}$ used in a proof is needed for another proof, we write it with an index or a subscript, as $C_{2}$ or $C_{*}$ for instance.

2.2. Differential operators and PDEs. If $f:[0, T] \times \mathbb{R}^{d} \rightarrow \mathbb{R}$ is a smooth function, we set $\partial_{j} f=\frac{\partial f}{\partial x_{j}}$ and $\partial_{t} f=\frac{\partial f}{\partial t}$. We denote by $\nabla f=\left(\partial_{i} f\right)_{1 \leq i \leq d}$ the gradient of $f$ and by $\Delta f=$ $\sum_{j} \partial_{j j}^{2} f$ its Laplacian. More generally, for a given set of non negative definite matrixes $a(t, x)=$ $\sigma(t, x) \sigma^{*}(t, x):[0, T] \times \mathbb{R}^{d} \rightarrow \mathbb{R}^{d} \otimes \mathbb{R}^{d}$, we set

$$
\Delta_{\sigma} f(t, x)=\sum_{i, j} a_{i j}(t, x) \partial_{i j}^{2} f(t, x) .
$$

For a smooth vector field $\Phi:[0, T] \times \mathbb{R}^{d} \rightarrow \mathbb{R}^{d}$, we denote by $\Phi^{j}$ its $j^{\text {th }}$-component, by $\nabla \Phi$ its differential with respect to $x$, that we represent into the canonical basis of $\mathbb{R}^{d}: \nabla \Phi=\left(\partial_{j} \Phi^{i}\right)_{i, j}$, and 
by $\nabla \cdot \Phi=\sum_{j} \partial_{j} \Phi^{j}$ its divergence. As usual, $\Delta \Phi$ denotes the vector $\left(\Delta \Phi^{j}\right)_{j}$. The notation $(\Psi \cdot \nabla) \Phi$ denotes the parallel derivative of $\Phi$ along $\Psi$, whose coordinates are: $((\Psi \cdot \nabla) \Phi)^{i}=\sum_{j} \Psi^{j} \partial_{j} \Phi^{i}$.

Throughout the paper, we consider parabolic operators whose generic form is given by

$$
\mathcal{L}_{b, \sigma}=\partial_{t}+b(t, x) \cdot \nabla+\frac{1}{2} \Delta_{\sigma}
$$

where $b:[0, T] \times \mathbb{R}^{d} \rightarrow \mathbb{R}^{d}$ and $\sigma:[0, T] \times \mathbb{R}^{d} \rightarrow \mathbb{R}^{d} \otimes \mathbb{R}^{d}$.

2.3. Probability space and Diffusions. We are given a filtered probability space $\left(\Omega, \mathcal{F},\left(\mathcal{F}_{t}\right), \mathbb{P}\right)$ on which a standard $d$-dimensional $\left(\mathcal{F}_{t}\right)$-adapted Brownian motion $W(\cdot)$ is defined. A diffusion starting from $x$ at time $t$ is denoted by $X_{t, x}$ and reads:

$$
X_{t, x}(s)=x+\int_{t}^{s} b\left(r, X_{t, x}(r)\right) d r+\int_{t}^{s} \sigma\left(r, X_{t, x}(r)\right) d W_{t, 0}(r), \quad 0 \leq t \leq s \leq T,
$$

where $b:[0, T] \times \mathbb{R}^{d} \rightarrow \mathbb{R}^{d}, \sigma:[0, T] \times \mathbb{R}^{d} \rightarrow \mathbb{R}^{d} \otimes \mathbb{R}^{d}$ and:

$$
W_{t, 0}(s)=W(s)-W(t) .
$$

More generally, under suitable conditions on $\sigma, W_{t, x}^{\sigma}$ denotes the unique strong solution of the SDE

$$
W_{t, x}^{\sigma}(r)=x+\int_{t}^{r} \sigma\left(u, W_{t, x}^{\sigma}(u)\right) d W_{t, 0}(u) \quad 0 \leq t \leq r \leq T .
$$

\section{StUdy of a Class of PARABolic QUASI-LinEar PDES}

3.1. Assumptions and preliminaries lemmas. To establish the main results of this paper we introduce the following assumptions:

$(\mathbf{H})$ A function $h:[0, T] \times \mathbb{R}^{d} \times \mathbb{R}^{d} \rightarrow \mathbb{R}^{d}$ satisfies Assumption $(H)$ if there exist $\left(c_{1}, c_{2}, c_{3}\right) \in$ $\mathbb{R}_{+}^{3}$, a non negative function $M \in L^{1}\left([0, T], L^{\infty}\left(\mathbb{R}^{d}\right)\right)$ and a function $\varphi: \mathbb{R}^{d} \rightarrow \mathbb{R}$ bounded on all compact sets such that for all $(t, x, y, z) \in[0, T] \times \mathbb{R}^{d} \times \mathbb{R}^{d} \times \mathbb{R}^{d}$ :

$$
\begin{aligned}
|h(t, x, y)| & \leq M(t, x)+c_{1}|y|, \\
|h(t, x, y)-h(t, x, z)| & \leq c_{2}|y-z|, \\
\left|\nabla_{x} h(t, x, y)\right|+\left|\nabla_{x}^{2} h(t, x, y)\right| & \leq c_{3}+\varphi(y) .
\end{aligned}
$$

We set $c_{M}:=\|M\|_{L^{1}\left([0, T], L^{\infty}\left(\mathbb{R}^{d}\right)\right)}$.

$\left(\mathbf{H}_{\boldsymbol{\Delta}}\right)$ A map $\sigma:[0, T] \times \mathbb{R}^{d} \rightarrow \mathbb{R}^{d} \otimes \mathbb{R}^{d}$ satisfies Assumption $\left(H_{\Delta}\right)$ if $\sigma \in C^{\infty}$ and

$$
\begin{aligned}
|\sigma(t, x) \xi|^{2} & \geq \mu|\xi|^{2}, \quad \xi \in \mathbb{R}^{d}, \\
\|\sigma\|_{\infty}+\|\nabla \sigma\|_{\infty} & <\infty \\
\left\|\sigma^{-1}\right\|_{\infty}+\left\|\nabla \sigma^{-1}\right\|_{\infty}+\left\|\nabla^{2} \sigma^{-1}\right\|_{\infty} & <\infty .
\end{aligned}
$$

$\left(\mathbf{H}_{\boldsymbol{\Phi}}\right)$ A map $\phi:(t, x, y) \in[0, T] \times \mathbb{R}^{d} \times \mathbb{R}^{d} \rightarrow \mathbb{R}^{d}$ satisfies Assumption $\left(H_{\Phi}\right)$ if $\phi \in C^{\infty}, \phi(\cdot, \cdot, 0)$ is bounded on $[0, T] \times \mathbb{R}^{d}$, and if

$$
\nabla_{x} \phi, \quad \nabla_{y} \phi, \quad \nabla_{x, x}^{2} \phi, \quad \nabla_{x, y}^{2} \phi, \quad \nabla_{y, y}^{2} \phi
$$

are bounded in $y$ on any compact subset $\mathcal{K} \in \mathbb{R}^{d}$ uniformly in $(t, x)$ on $[0, T] \times \mathbb{R}^{d}$. 
If $\phi$ satisfies Assumption $\left(H_{\Phi}\right)$, let us define the following increasing functions:

$$
\begin{aligned}
\Phi_{1}^{1}(\rho) & =\sup _{t \in[0, T], x \in \mathbb{R}^{d},|y| \leq \rho}\left|\nabla_{x} \phi(t, x, y)\right|, \\
\Phi_{2}^{1}(\rho) & =\sup _{t \in[0, T], x \in \mathbb{R}^{d},|y| \leq \rho}\left|\nabla_{y} \phi(t, x, y)\right|, \quad \rho \geq 0 .
\end{aligned}
$$

Remark 1. Hypothesis $\left(H_{\Phi}\right)$ obviously includes the case where $\sigma=C \mathrm{Id}, \phi=b(t, x)+y^{\alpha}$ where $\alpha$ is a multi index and $b$ is a bounded function.

We will use several times the following simple lemmas:

Lemma 3.1. Let $\left(g_{n}:[0, T] \rightarrow \mathbb{R}\right)_{n \in \mathbb{N}}$ be a sequence of non negative measurable functions. Let $\theta \in L^{1}\left([0, T], \mathbb{R}^{+}\right)$. If for all $n \geq 0$ and $t \in[0, T]$

$$
g_{n+1}(t) \leq K_{0}+K_{1} \int_{t}^{T} g_{n}(s) d s+\int_{t}^{T} \theta(s) d s, \quad g_{0}(t) \leq \widetilde{K}_{0}
$$

then for all $t \in[0, T]$

$$
g_{n}(t) \leq K_{0} \sum_{k=0}^{n-1} \frac{\left(K_{1}(T-t)\right)^{k}}{k !}+\int_{0}^{T} \theta(s) d s \sum_{k=1}^{n-1} \frac{\left(K_{1}(T-t)\right)^{k}}{k !}+\widetilde{K}_{0} \frac{\left(K_{1}(T-t)\right)^{n}}{n !}+\int_{t}^{T} \theta(s) d s .
$$

We also have the useful expression:

$$
g_{n}(t) \leq\left(\max \left(K_{0}, \widetilde{K}_{0}\right)+\int_{0}^{T} \theta(s) d s\right) e^{K_{1}(T-t)}-\int_{0}^{t} \theta(s) d s .
$$

Proof. The proof stems from an immediate induction.

Lemma 3.2. Let $\phi$ satisfy Assumption $\left(H_{\Phi}\right)$. Let $\chi:[0, T] \times \mathbb{R}^{d} \rightarrow \mathbb{R}^{d}$ be a bounded $C^{2}$ vector field with bounded derivatives. Then

$$
\begin{aligned}
& \sup _{t \in[0, T], x \in \mathbb{R}^{d}}|\phi(t, x, \chi(t, x))| \leq\|\phi(\cdot, \cdot, 0)\|_{\infty}+\Phi_{2}^{1}\left(\|\chi\|_{\infty}\right)\|\chi\|_{\infty} \\
& \sup _{x \in \mathbb{R}^{d}}\left|\frac{\partial}{\partial x}(\phi(t, x, \chi(t, x)))\right| \leq \Phi_{1}^{1}\left(\|\chi\|_{\infty}\right)+\Phi_{2}^{1}\left(\|\chi\|_{\infty}\right)\|\nabla \chi(t)\|_{\infty} \\
& \sup _{x \in \mathbb{R}^{d}}\left|\frac{\partial^{2}}{\partial x^{2}}(\phi(t, x, \chi(t, x)))\right| \leq C+C\|\nabla \chi(t)\|_{\infty}^{2}+C\left\|\nabla^{2} \chi(t)\right\|_{\infty} .
\end{aligned}
$$

Proof. The first inequality comes from the inequality

$$
|\phi(t, x, \chi(t, x))-\phi(t, x, 0)| \leq \sup _{y \in B\left(0,\|\chi\|_{\infty}\right)}\left|\nabla_{y} \phi(t, x, y)\right||\chi(t, x)|,
$$

and Assumption $\left(H_{\Phi}\right)$. The Leibniz rule and Assumption $\left(H_{\Phi}\right)$ yield the two other ones.

Lemma 3.3. The mapping $x \mapsto W_{t, x}^{\sigma}(r)$ is differentiable for all $x \in \mathbb{R}^{d}$ a.s. Moreover its differential satisfies the SDE

$$
\nabla W_{t, x}^{\sigma}(r)=\mathrm{Id}+\int_{t}^{r} \nabla \sigma\left(u, W_{t, x}^{\sigma}(u)\right) \nabla W_{t, x}^{\sigma}(u) d W_{t, 0}(u) .
$$


For all $x, y \in \mathbb{R}^{d}$ and all $p \geq 1$, we have the followings bounds:

$$
\begin{aligned}
E\left|W_{t, y}^{\sigma}(s)-W_{t, x}^{\sigma}(s)\right|^{2 p} & \leq C_{p}|y-x|^{2 p}, \\
E\left|\nabla W_{t, x}^{\sigma}(r)\right|^{2 p} & \leq C_{p}, \\
E\left|\nabla W_{t, y}^{\sigma}(s)-\nabla W_{t, x}^{\sigma}(s)\right|^{2 p} & \leq C_{p}|y-x|^{2 p} .
\end{aligned}
$$

Proof. The Gronwall Lemma and (3.47) yield the inequality (3.28).

From (3.47), we can write (3.27) (cf e.g. [11]). Using the assumption $\|\nabla \sigma\|_{\infty}<\infty$, the Burkolder-Davis-Gundy (BDG) inequality and the Gronwall lemma, we obtain (3.29). Using (3.27-3.29-3.28), we can finally prove (3.30).

3.2. Main Result. We consider the following Cauchy problem:

$$
\left\{\begin{aligned}
\partial_{t} v(t, x)+(\phi(t, x, v) \cdot \nabla) v(t, x)-\frac{1}{2} \Delta_{\nu} v(t, x) & =h(t, x, v(t, x)) \\
v(0, x) & =v_{0}(x)
\end{aligned}\right.
$$

where $\phi:[0, T] \times \mathbb{R}^{d} \times \mathbb{R}^{d} \rightarrow \mathbb{R}^{d}, \nu:[0, T] \times \mathbb{R}^{d} \rightarrow \mathbb{R}^{d} \otimes \mathbb{R}^{d}, h:[0, T] \times \mathbb{R}^{d} \times \mathbb{R}^{d} \rightarrow \mathbb{R}^{d}, v_{0}: \mathbb{R}^{d} \rightarrow \mathbb{R}^{d}$ are given while $v:[0, T] \times \mathbb{R}^{d} \rightarrow \mathbb{R}^{d}$ is the unknown.

For all $t \in[0, T]$, let

$$
\lambda_{1}(t)=\left(\left\|v_{0}\right\|_{\infty}+\int_{0}^{T}\|M(s)\|_{\infty} d s\right) e^{c_{1} t}-\int_{t}^{T}\|M(s)\|_{\infty} d s .
$$

We then define the following constants:

$$
\begin{aligned}
\lambda_{1} & :=\lambda_{1}(T) \\
\lambda_{2} & :=\left\|\sigma^{-1}\right\|_{\infty}^{2}\left(\|\phi(\cdot, \cdot, 0)\|_{\infty}+\Phi_{2}^{1}\left(\lambda_{1}\right)\right)^{2} \\
\lambda_{3} & :=4\left\|E J^{\sigma}\right\|_{\infty}^{2} e^{2 \lambda_{2} T},
\end{aligned}
$$

where $\sigma(t, x)=\nu(T-t,-x), \phi$ satisfies $\left(H_{\Phi}\right)$, and $J_{t, x}^{\sigma}(s)$ denotes the module of the Jacobian of the inverse of the $C^{1}$ diffeomorphism $x \mapsto W_{t, x}^{\sigma}(s)$.

The main result of this section is the following theorem.

Theorem 3.4. Fix $T>0$. Let $\phi$ satisfy Assumption $\left(H_{\Phi}\right), v_{0} \in C_{b, 2}^{\infty}\left(\mathbb{R}^{d}\right)$, and let $h:[0, T] \times$ $\mathbb{R}^{d} \times \mathbb{R}^{d} \rightarrow \mathbb{R}^{d}$ be a $C^{\infty}$ function satisfying Assumption $(H)$. Let $\nu:[0, T] \times \mathbb{R}^{d} \rightarrow \mathbb{R}^{d} \otimes \mathbb{R}^{d}$ verify Assumption $\left(H_{\Delta}\right)$. Then there exists a unique bounded solution $v \in C^{\infty}\left([0, T] \times \mathbb{R}^{d}\right)$ verifying the Cauchy problem (3.31). The scheme (1.6-1.7) uniformly converges to $v$ with the rate

$$
\left\|v-v_{N}\right\|_{\infty} \leq \sum_{n \geq N} \sqrt{\frac{(C T)^{n}}{n !}}
$$

where $C>0$ only depends on the data of the equation.

Moreover, the following bound holds for all $t \in[0, T]:\|v(t)\|_{\infty} \leq \lambda_{1}(t)$.

In addition, if $v_{0} \in L^{2}\left(\mathbb{R}^{d}\right) \cap C_{b, 2}^{\infty}\left(\mathbb{R}^{d}\right)$ and $M \in L^{2}\left([0, T] \times \mathbb{R}^{d}\right)$ then

$$
\|v(t)\|_{2}^{2} \leq\left(\lambda_{3}\left\|v_{0}\right\|_{2}^{2}+\int_{0}^{T} \lambda_{3}\|M(s)\|_{2}^{2} d s\right) e^{\lambda_{3} c_{1}^{2} T t}-\int_{t}^{T} \lambda_{3}\|M(s)\|_{2}^{2} d s .
$$


Proof. The proof is based on the following steps. First, using the associated backward Cauchy system we define a sequence $\left(u_{n}\right)$ derived from a Feynman-Kac representation. In Step 1, we show the uniform convergence of $\left(u_{n}\right)$ using Girsanov's transformation and Gronwall's Lemma. This proof involves estimates on Girsanov's densities which are a slight generalization of those already obtained by Busnello [5]. In Step 2, we obtain uniform bounds of the derivatives of $u_{n}$. In Step 3, we conclude that the limit $u$ of $\left(u_{n}\right)$ is a smooth solution of the Cauchy system by using the previous results and applying a Friedman's Theorem (Th. 15 p.80 in [8]) on uniform parabolic PDEs. In Step 4, we prove the uniqueness of the solution and eventually in Step 5, we obtain suitable bounds in $L^{2}$ for this solution.

Using the change of variable $u(t, x)=v(T-t,-x)$, we turn the initial Cauchy system into the following backward one:

$$
\left\{\begin{aligned}
\partial_{t} u(t, x)+(\phi(t, x, u) \cdot \nabla) u(t, x)+\frac{1}{2} \Delta_{\sigma} u(t, x) & =-g(t, x, u(t, x)) \\
u(T, x) & =u_{0}(x),
\end{aligned}\right.
$$

where $u_{0}:=v_{0}, g(t, x, y)=h(T-t,-x, y)$ and $\sigma(t, x)=\nu(T-t,-x)$. In particular, $g$ and $\sigma$ satisfy the same conditions than $h$ and $\nu$ respectively.

Step 0. Definition of a recursive scheme.

Let us define by induction the sequence $u_{n}:[0, T] \times \mathbb{R}^{d} \rightarrow \mathbb{R}^{d}, n \geq 0$, as follows.

Let $u_{0}(t, x)=u_{0}(x)$ for all $(t, x) \in[0, T] \times \mathbb{R}^{d}$. Given $u_{n} \in C^{2}\left([0, T] \times \mathbb{R}^{d}\right)$, we define $u_{n+1}$ as the solution of the following PDE:

$$
\left\{\begin{aligned}
\partial_{t} u_{n+1}(t, x)+\left(w_{n} \cdot \nabla\right) u_{n+1}(t, x)+\frac{1}{2} \Delta_{\sigma} u_{n+1}(t, x) & =f_{n}(t, x) \\
u_{n+1}(T, x) & =u_{0}(x),
\end{aligned}\right.
$$

where

$$
\begin{aligned}
w_{n}(t, x) & =\phi\left(t, x, u_{n}(t, x)\right) \\
f_{n}(t, x) & =g\left(t, x, u_{n}(t, x)\right) .
\end{aligned}
$$

The solution of this linear parabolic PDE can be represented by means of the Feynman-Kac formula :

$$
u_{n+1}(t, x)=E\left[\int_{t}^{T} f_{n}\left(s, X_{t, x}^{(n)}(s)\right) d s+u_{0}\left(X_{t, x}^{(n)}(T)\right)\right]
$$

where $X_{t, x}^{(n)}$ is the stochastic flow defined by:

$$
X_{t, x}^{(n)}(s)=x+\int_{t}^{s} w_{n}\left(r, X_{t, x}^{(n)}(r)\right) d r+\int_{t}^{s} \sigma\left(r, X_{t, x}^{(n)}(r)\right) d W_{t, 0}(r), \quad 0 \leq t \leq s \leq T .
$$

If $u_{0} \in C_{b, 2}^{2}\left(\mathbb{R}^{d}\right)$, one can show by induction that

$$
\begin{aligned}
\left\|u_{n}\right\|_{\infty} & <\infty \\
\left\|\nabla u_{n}\right\|_{\infty} & <\infty
\end{aligned}
$$


using for instance Eq. (5.20) and (5.21) p.148 in [9] (letting $a \rightarrow 0$ ). Besides, by virtue of (3.25) we obtain that $w_{n}$ is Lipschitz in $x$ uniformly in $t$, so that the diffusion $X_{t, x}^{(n)}$ is well defined. As a conclusion, the scheme is well defined and its Feynman-Kac representation holds.

Finally, from Theorem 10 p. 72 in [8], we deduce by induction that $u_{n} \in C^{2}\left([0, T] \times \mathbb{R}^{d}\right)$.

Step 1. Convergence of $\left(u_{n}\right)_{n \in \mathbb{N}}$ in $L^{\infty}\left([0, T] \times \mathbb{R}^{d}\right)$.

The expression (3.40) shows that the sequence $\left(u_{n}\right)_{n \geq 0}$ is bounded in $L^{\infty}\left([0, T] \times \mathbb{R}^{d}\right)$. Indeed, due to Assumption $(H)$, we can write:

$$
\left\|u_{n+1}(t)\right\|_{\infty} \leq\left\|u_{0}\right\|_{\infty}+\int_{t}^{T}\|M(s)\|_{\infty} d s+c_{1} \int_{t}^{T}\left\|u_{n}(s)\right\|_{\infty} d s
$$

and we then apply Lemma 3.1 with $K_{0}=\widetilde{K}_{0}=\left\|u_{0}\right\|_{\infty}, K_{1}=c_{1}$ and $\theta(s)=\|M(s)\|_{\infty}$ to obtain for all $t \in[0, T]$ the bound:

$$
\sup _{n \geq 0}\left\|u_{n+1}(t)\right\|_{\infty} \leq\left(\left\|u_{0}\right\|_{\infty}+\int_{0}^{T}\|M(s)\|_{\infty} d s\right) e^{c_{1}(T-t)}-\int_{0}^{t}\|M(s)\|_{\infty} d s \leq \lambda_{1} .
$$

A Girsanov transformation yields the following expression for $u_{n+1}$ :

$$
u_{n+1}(t, x)=E\left[\left(\int_{t}^{T} f_{n}\left(s, W_{t, x}^{\sigma}(s)\right) d s+u_{0}\left(W_{t, x}^{\sigma}(T)\right)\right) Z_{t, T}^{(x, n)}\right]
$$

where

$$
Z_{t, s}^{(x, n)}=\exp \left\{\int_{t}^{s}\left(\sigma^{-1} w_{n}\right)\left(r, W_{t, x}^{\sigma}(r)\right) d W_{t, 0}(r)-\frac{1}{2} \int_{t}^{s}\left|\sigma^{-1} w_{n}\right|^{2}\left(r, W_{t, x}^{\sigma}(r)\right) d r\right\}
$$

and $W_{t, x}^{\sigma}$ is the solution of the SDE

$$
W_{t, x}^{\sigma}(r)=x+\int_{t}^{r} \sigma\left(u, W_{t, x}^{\sigma}(u)\right) d W_{t, 0}(u) \quad 0 \leq t \leq r \leq T .
$$

Indeed, under $\mathbb{Q}:=Z_{t, T}^{(x, n)} \cdot \mathbb{P}$, the process

$$
s \mapsto \widetilde{W}_{t, 0}(s):=W_{t, 0}(s)-\int_{t}^{s}\left(\sigma^{-1} w_{n}\right)\left(r, W_{t, x}^{\sigma}(r)\right) d r
$$

is a Brownian motion. We then write:

$$
d W_{t, x}^{\sigma}(r)=w_{n}\left(r, W_{t, x}^{\sigma}(r)\right) d r+\sigma\left(r, W_{t, x}^{\sigma}(r)\right) d \widetilde{W}_{t, 0}(r) .
$$

It follows that $W_{t, x}^{\sigma}$ is a weak solution of the SDE satisfied by $X_{t, x}^{(n)}$. So $W_{t, x}^{\sigma}$ has the same law under $\mathbb{Q}$ than $X_{t, x}^{(n)}$ under $\mathbb{P}$, and we deduce (3.45).

Lemma 3.5. The sequence $\left(u_{n}\right)$ defined by (3.40 - 3.39) uniformly converges on $[0, T] \times \mathbb{R}^{d}$.

Proof. We study the convergence of $\left(u_{n}\right)$ via the series $\sum \gamma_{n}$ where

$$
\gamma_{n}(t)=\left\|\left(u_{n+1}-u_{n}\right)(t)\right\|_{\infty} .
$$

Let us now remark that the martingale $s \mapsto Z_{t, s}^{(x, n)}$ solves the SDE

$$
Z_{t, s}^{(x, n)}=1+\int_{t}^{s}\left(\sigma^{-1} w_{n}\right)\left(r, W_{t, x}^{\sigma}(r)\right) Z_{t, r}^{(x, n)} d W_{t, 0}(r) .
$$


From (3.24) we deduce that $\left(w_{n}\right)_{n \geq 0}$ is bounded in $L^{\infty}\left([0, T] \times \mathbb{R}^{d}\right)$ since $\left(u_{n}\right)_{n \geq 0}$ is bounded in $L^{\infty}\left([0, T] \times \mathbb{R}^{d}\right)$.

Moreover, the Ito isometry and the Gronwall lemma imply

$$
E\left(Z_{t, s}^{(x, n)}\right)^{2} \leq \exp \left(2\left\|\sigma^{-1}\right\|_{\infty}^{2}(s-t)\left\|w_{n}\right\|_{\infty}^{2}\right), \quad t \leq s
$$

Inequality (3.24) applied with $\chi=u_{n}$, and $\sup _{n \geq 0}\left\|u_{n}\right\|_{\infty}<\infty$ then yield

$$
\sup _{x \in \mathbb{R}^{d}, t, s \in[0, T], n \geq 0} E\left(Z_{t, s}^{(x, n)}\right)^{2}<\infty .
$$

Set $0<T^{\prime} \leq T$. Recall that $w_{n+1}-w_{n}=\phi\left(t, x, u_{n+1}\right)-\phi\left(t, x, u_{n}\right)$. Since $\nabla_{y} \phi$ is bounded on $[0, T] \times \mathbb{R}^{d} \times B\left(0, \lambda_{1}\right)$, we obtain the following inequalities:

$$
\begin{aligned}
E\left|Z_{t, T^{\prime}}^{(x, n+1)}-Z_{t, T^{\prime}}^{(x, n)}\right|^{2} \leq & C E\left|\int_{t}^{T^{\prime}}\left(w_{n+1}-w_{n}\right)\left(r, W_{t, x}^{\sigma}(r)\right) Z_{t, r}^{(x, n+1)} d W_{t, 0}(r)\right|^{2} \\
& +C E\left|\int_{t}^{T^{\prime}} w_{n}\left(r, W_{t, x}^{\sigma}(r)\right)\left(Z_{t, r}^{(x, n+1)}-Z_{t, r}^{(x, n)}\right) d W_{t, 0}(r)\right|^{2} \\
\leq & C E \int_{t}^{T}\left|\left(u_{n+1}-u_{n}\right)\left(r, W_{t, x}^{\sigma}(r)\right)\right|^{2}\left|Z_{t, r}^{(x, n+1)}\right|^{2} d r \\
& +C E \int_{t}^{T^{\prime}}\left|w_{n}\left(r, W_{t, x}^{\sigma}(r)\right)\right|^{2}\left|Z_{t, r}^{(x, n+1)}-Z_{t, r}^{(x, n)}\right|^{2} d r \\
\leq & C \int_{t}^{T} \gamma_{n}(t)^{2} d r+C E \int_{t}^{T^{\prime}} E\left|Z_{t, r}^{(x, n+1)}-Z_{t, r}^{(x, n)}\right|^{2} d r .
\end{aligned}
$$

The Gronwall Lemma (making $T^{\prime}$ vary) then yields:

$$
E\left|Z_{t, T^{\prime}}^{(x, n+1)}-Z_{t, T^{\prime}}^{(x, n)}\right|^{2} \leq C \int_{t}^{T} \gamma_{n}(t)^{2} d r .
$$

We write

$$
\begin{array}{rl}
\left(u_{n+1}-u_{n}\right)(t, x)=E & E\left[\left(\int_{t}^{T}\left(f_{n}\left(s, W_{t, x}^{\sigma}(s)\right)-f_{n-1}\left(s, W_{t, x}^{\sigma}(s)\right)\right) d s\right) Z_{t, T}^{(x, n)}\right] \\
+ & E\left[\left(\int_{t}^{T} f_{n-1}\left(s, W_{t, x}^{\sigma}(s)\right) d s\right)\left(Z_{t, T}^{(x, n)}-Z_{t, T}^{(x, n-1)}\right)\right] \\
+ & E\left[u_{0}\left(W_{t, x}^{\sigma}(T)\right)\left(Z_{t, T}^{(x, n)}-Z_{t, T}^{(x, n-1)}\right)\right] .
\end{array}
$$

Due to the Jensen and Cauchy Schwarz inequalities, the Lipschitz condition satisfied by $g$, Inequalities (3.59) and (3.52) with $T=T^{\prime}$, we obtain:

$$
\gamma_{n}(t)^{2} \leq C \int_{t}^{T} \gamma_{n-1}(r)^{2} d r
$$

Lemma 3.1 then implies:

$$
\gamma_{n}(t)^{2} \leq C^{n+1} \frac{(T-t)^{n}}{n !}
$$


Therefore, since the serie $\sum \sqrt{\frac{(C(T-t))^{n}}{n !}}$ is convergent, it follows

$$
\sum_{n \geq 0} \sup _{t, x}\left|\left(u_{n+1}-u_{n}\right)(t, x)\right|<\infty .
$$

This yields the conclusion of Lemma 3.5.

Step 2. : Bounds for the derivatives of $\left(u_{n}\right)$.

Lemma 3.6. The map

$$
\left\{\begin{aligned}
\mathbb{R}^{d} & \longrightarrow L^{2}(\Omega) \\
x & \longmapsto Z_{t, s}^{(x, n)}
\end{aligned}\right.
$$

is differentiable for all $x \in \mathbb{R}^{d}$. Moreover, its $L^{2}(\Omega)$-valued derivative reads $\nabla Z_{t, s}^{(x, n)}=Z_{t, s}^{(x, n)} \nabla Y_{t, s}^{(x, n)}$ where

$$
Y_{t, s}^{(x, n)}:=\int_{t}^{s} r_{n}\left(r, W_{t, x}^{\sigma}(r)\right) d W_{t, 0}(r)-\frac{1}{2} \int_{t}^{s} r_{n}^{2}\left(r, W_{t, x}^{\sigma}(r)\right) d s .
$$

Proof. Set

$$
r_{n}=\sigma^{-1} w_{n} .
$$

Recall that $\sup _{n \geq 0}\left\|u_{n}\right\|_{\infty}<\infty$ and

$$
\sup _{x \in \mathbb{R}^{d}, t, s \in[0, T], n \geq 0} E\left(Z_{t, s}^{(x, n)}\right)^{2}<\infty,
$$

and more generally from the Doleans form of $Z$, we can prove that for all $p \in \mathbb{Z}$ there exists some constants $C_{p}$ independent of $n$ such that

$$
\sup _{x \in \mathbb{R}^{d}, t, s \in[0, T], n \geq 0} E\left(Z_{t, s}^{(x, n)}\right)^{2 p} \leq C_{p} .
$$

In addition, let us note that if $\alpha_{s} \in[0,1]$ is a $\mathcal{F}_{s}$-adapted random variable then, from the inequality $\left(Z_{t, s}^{(x, n)}\right)^{2 p\left(1-\alpha_{s}\right)} \leq 1+\left(Z_{t, s}^{(x, n)}\right)^{2 p}$, we can choose $C_{p}$ such that

$$
\sup _{x \in \mathbb{R}^{d}, t, s \in[0, T], n \geq 0} E\left(Z_{t, s}^{(x, n)}\right)^{2 p\left(1-\alpha_{s}\right)} \leq C_{p}, \quad p \in \mathbb{Z} .
$$

We claim that the map

$$
\left\{\begin{aligned}
\mathbb{R}^{d} & \longrightarrow L^{2 p}(\Omega) \\
x & \longmapsto Y_{t, s}^{(x, n)}
\end{aligned}\right.
$$

is differentiable everywhere on $\mathbb{R}^{d}$, and for any $p \geq 1$. Moreover its derivative $\nabla Y_{t, s}^{\left(x_{0}, n\right)}$ at $x_{0}$ is computed by differentiating (3.57) under the integral sign. Indeed, recall that $\sup _{[0, T]}\left\|\nabla u_{n}(t)\right\|_{\infty}<$ $\infty$; Hence from Assumption $\left(H_{\Delta}\right)$ and Lemma 3.2, we first obtain that

$$
\begin{aligned}
\left\|w_{n}\right\|_{\infty}+\left\|r_{n}\right\|_{\infty} & <\infty \\
\left\|\nabla w_{n}(s)\right\|_{\infty} & <C+C\left\|\nabla u_{n}(s)\right\|_{\infty}, \\
\left\|\nabla r_{n}(s)\right\|_{\infty} & <C+C\left\|\nabla u_{n}(s)\right\|_{\infty}, \quad 0 \leq s \leq T .
\end{aligned}
$$


Then, we use the BDG inequality to show that there exist some constants $C_{p}$ such that

$$
E\left|Y_{t, s}^{(x, n)}-Y_{t, s}^{\left(x_{0}, n\right)}-\nabla Y_{t, s}^{\left(x_{0}, n\right)}\left(x-x_{0}\right)\right|^{2 p} \leq C_{p}\left|x-x_{0}\right|^{2 p}, \quad p \geq 1
$$

and we apply the dominated convergence theorem to conclude about the desired differentiability.

Now, recall that $Z_{t, s}^{(x, n)}=e^{Y_{t, s}^{(x, n)}}$. By virtue of measurable selection arguments there is a $\mathcal{F}_{T}$-measurable random variable $\alpha_{s} \in[0,1]$ such that

$$
\begin{aligned}
& E\left|Z_{t, s}^{(x, n)}-Z_{t}^{\left(x_{0}, n\right)}(s)-Z_{t}^{\left(x_{0}, n\right)}(s) \nabla Y_{t, s}^{\left(x_{0}, n\right)}\left(x-x_{0}\right)\right|^{2} \\
& =E\left|\frac{e^{Y_{t, s}^{(x, n)}}-e^{Y_{t, s}^{\left(x_{0}, n\right)}}}{Y_{t, s}^{(x, n)}-Y_{t, s}^{\left(x_{0}, n\right)}}\left(Y_{t, s}^{(x, n)}-Y_{t, s}^{\left(x_{0}, n\right)}\right)-Z_{t}^{\left(x_{0}, n\right)}(s) \nabla Y_{t, s}^{\left(x_{0}, n\right)}\left(x-x_{0}\right)\right|^{2} \\
& =E\left|e^{\alpha_{s} Y_{t, s}^{\left(x_{0}, n\right)}+\left(1-\alpha_{s}\right) Y_{t, s}^{(x, n)}}\left(Y_{t, s}^{(x, n)}-Y_{t, s}^{\left(x_{0}, n\right)}\right)-Z_{t}^{\left(x_{0}, n\right)}(s) \nabla Y_{t, s}^{\left(x_{0}, n\right)}\left(x-x_{0}\right)\right|^{2} \\
& \leq 2 E\left|\left(e^{\alpha_{s} Y_{t, s}^{\left(x_{0}, n\right)}+\left(1-\alpha_{s}\right) Y_{t, s}^{(x, n)}}-Z_{t}^{\left(x_{0}, n\right)}(s)\right) Y_{t, s}^{(x, n)}-Y_{t, s}^{\left(x_{0}, n\right)}\right|^{2} \\
& +2 E\left|Z_{t}^{\left(x_{0}, n\right)}(s)\left(Y_{t, s}^{(x, n)}-Y_{t, s}^{\left(x_{0}, n\right)}-\nabla Y_{t, s}^{\left(x_{0}, n\right)}\left(x-x_{0}\right)\right)\right|^{2}:=A(x)+B(x) .
\end{aligned}
$$

Let $\epsilon>0$. Using the Cauchy-Schwarz inequality and (3.66), we then bound $A(x)$ by the square root of

$$
\begin{aligned}
& E\left|e^{\left(1-\alpha_{s}\right)\left(Y_{t, s}^{(x, n)}-Y_{t, s}^{\left(x_{0}, n\right)}\right)}-1\right|^{4} E\left|Z_{t}^{\left(x_{0}, n\right)}(s)\left(Y_{t, s}^{(x, n)}-Y_{t, s}^{\left(x_{0}, n\right)}\right)\right|^{4} \\
& \quad \leq C\left|x-x_{0}\right|^{4}\left(\epsilon+\sqrt{\left.E\left|e^{\left(1-\alpha_{s}\right)\left(Y_{t, s}^{(x, n)}-Y_{t, s}^{\left(x_{0}, n\right)}\right)}-1\right| \sqrt[8]{P\left(\left|Y_{t, s}^{(x, n)}-Y_{t, s}^{\left(x_{0}, n\right)}\right|>\eta\right)}\right)},\right.
\end{aligned}
$$

where $\eta>0$ is such that $\left(e^{z}-1\right)^{4} \leq \epsilon$ for $|z| \leq \eta$. From (3.61), we deduce that $A(x)=o\left(\left|x-x_{0}\right|^{2}\right)$. Finally, we use the Cauchy-Schwarz inequality and the differentiability of $x \mapsto Y_{t, s}^{(x, n)} \in L^{4}(\Omega)$ to conclude that $B(x)=o\left(\left|x-x_{0}\right|^{2}\right)$.

Lemma 3.7. The sequence $\left(u_{n}\right)$ defined by (3.40 - 3.39) verifies

$$
\sup _{n \geq 0}\left\|\nabla u_{n}\right\|_{\infty}<\infty .
$$

Proof. We prove the following statement: there exists a constant $C$ independent of $n$ such that

$$
\left\|\nabla u_{n+1}(t)\right\|_{\infty}^{2} \leq C+C \int_{t}^{T}\left\|\nabla u_{n}(r)\right\|_{\infty}^{2} d r
$$

In that case, since $\left\|\nabla u_{0}\right\|_{\infty}<\infty$, then for all $n \geq 0, \sup _{[0, T]}\left\|\nabla u_{n+1}(t)\right\|_{\infty}<\infty$. The sequence $\left(\nabla u_{n}\right)_{n \geq 0}$ is then uniformly bounded by virtue of Lemma 3.1. 
By virtue of the Itô integration by parts, we deduce:

$$
\nabla_{x} Z_{t, s}^{(x, n)}=\int_{t}^{s}\left(\nabla r_{n}\left(r, W_{t, x}^{\sigma}(r)\right) \nabla_{x} W_{t, x}^{\sigma}(r) Z_{t, r}^{(x, n)}+r_{n}\left(r, W_{t, x}^{\sigma}(r)\right) \nabla_{x} Z_{t, r}^{(x, n)}\right) d W_{t, 0}(r) .
$$

Thus

$$
E\left|\nabla Z_{t, s}^{(x, n)}\right|^{2}=\int_{t}^{s} E\left|\nabla r_{n}\left(r, W_{t, x}^{\sigma}(r)\right) \nabla_{x} W_{t, x}^{\sigma}(r) Z_{t, r}^{(x, n)}+r_{n}\left(r, W_{t, x}^{\sigma}(r)\right) \nabla Z_{t, r}^{(x, n)}\right|^{2} d r .
$$

Therefore, due to inequalities (3.60) and (3.29):

$$
E\left|\nabla Z_{t, s}^{(x, n)}\right|^{2} \leq C \int_{t}^{s}\left\|\nabla r_{n}(r)\right\|_{\infty}^{2} d r+C \int_{t}^{s} E\left|\nabla Z_{t, r}^{(x, n)}\right|^{2} d r
$$

Again, by Gronwall's Lemma and (3.63),

$$
E\left|\nabla Z_{t, s}^{(x, n)}\right|^{2} \leq C \int_{t}^{s}\left\|\nabla r_{n}(r)\right\|_{\infty}^{2} d r \leq C+C \int_{t}^{s}\left\|\nabla u_{n}(r)\right\|_{\infty}^{2} d r .
$$

We have:

$$
\nabla f_{n}(s, x)=\nabla_{x} g\left(s, x, u_{n}(s, x)\right)+\nabla_{y} g\left(s, x, u_{n}(s, x)\right) \nabla u_{n}(s, x),
$$

and then

$$
\left|\nabla f_{n}(s, x)\right| \leq C+C\left\|\nabla u_{n}(s)\right\|_{\infty} .
$$

Let us now compute the rate of increase

$$
\delta_{s}\left(u_{n+1}\right)\left(x, y_{\alpha}\right)=\frac{\left(u_{n+1}\left(s, y_{\alpha}\right)-u_{n+1}(s, x)\right)}{\alpha}
$$

of $\nabla u_{n+1}(s, \cdot)$ in any direction $e \in \mathbb{R}^{d},|e|=1$ at $x$ with $y_{\alpha}=x+\alpha e$ :

$$
\begin{aligned}
\delta_{t}\left(u_{n+1}\right)\left(x, y_{\alpha}\right)=E & {\left[\left(\int_{t}^{T} \delta_{s}\left(f_{n}\right)\left(W_{t, x}^{\sigma}(s), W_{t, y_{\alpha}}^{\sigma}(s)\right) d s+\delta\left(u_{0}\right)\left(W_{t, x}^{\sigma}(T), W_{t, y_{\alpha}}^{\sigma}(T)\right)\right) Z_{t, T}^{\left(y_{\alpha}, n\right)}\right] } \\
& +E\left[\left(\int_{t}^{T} f_{n}\left(s, W_{t, x}^{\sigma}(s)\right) d s+u_{0}\left(W_{t, x}^{\sigma}(T)\right)\right) \delta\left(Z_{t, T}^{(\cdot, n)}\right)\left(x, y_{\alpha}\right)\right]
\end{aligned}
$$

Using a Taylor expansion we write

$$
\begin{aligned}
&\left|\delta_{s}\left(f_{n}\right)\left(W_{t, x}^{\sigma}(s), W_{t, y_{\alpha}}^{\sigma}(s)\right)\right| \leq\left\|\nabla f_{n}(s)\right\|_{\infty}\left|W_{t, y_{\alpha}}(s)-W_{t, x}^{\sigma}(s)\right| \alpha^{-1} \\
&\left|\delta_{s}\left(u_{0}\right)\left(W_{t, x}^{\sigma}(s), W_{t, y_{\alpha}}^{\sigma}(s)\right)\right| \leq\left\|\nabla u_{0}(s)\right\|_{\infty}\left|W_{t, y_{\alpha}}(s)-W_{t, x}^{\sigma}(s)\right| \alpha^{-1} .
\end{aligned}
$$

Moreover,

$$
\delta\left(Z_{t, T}^{(\cdot, n)}\right)\left(x, y_{\alpha}\right) \underset{\alpha \rightarrow 0}{\stackrel{L^{2}(\Omega)}{\longrightarrow}} \nabla Z_{t, T}^{(x, n)} \cdot e
$$

Recall the inequality

$$
E\left|W_{t, y_{\alpha}}^{\sigma}(s)-W_{t, x}^{\sigma}(s)\right|^{2} \leq C\left|y_{\alpha}-x\right|^{2}=C \alpha^{2} .
$$

Then we apply the Cauchy-Schwarz inequality taking into account the uniform bound (3.73) in $x$, the uniform bound (3.59) in $x$ and $n$, the uniform bound (3.71) in $x$ and we get as $\alpha \rightarrow 0$ :

$$
\left|\nabla u_{n+1}(t, x)\right|^{2} \leq C+C \int_{t}^{T}\left\|\nabla u_{n}(r)\right\|_{\infty}^{2} d r
$$

where $C$ does not depend on $n$, which concludes the proof. 
Remark 2. Note that it is not possible to use directly the dominated convergence theorem to show the desired uniform bounds.

Lemma 3.8. The sequence $\left(u_{n}\right)$ defined by (3.40 - 3.39) verifies

$$
\sup _{n \geq 0}\left\|\nabla^{2} u_{n}\right\|_{\infty}<\infty
$$

Proof. We use the same method than the previous lemma. Recall that we have uniform bounds for $\left(r_{n}\right)$ in $n$ and $x$, but also for $\left(\nabla u_{n}\right)$ and then $\left(\nabla r_{n}\right)$ in $x$ and $n$ due to the previous lemma.

From (3.26), we deduce that

$$
\begin{aligned}
\left\|\nabla^{2} w_{n}(t)\right\|_{\infty} & \leq C+C\left\|\nabla u_{n}(t)\right\|_{\infty}^{2}+C\left\|\nabla^{2} u_{n}(t)\right\|_{\infty} \\
& \leq C+C\left\|\nabla^{2} u_{n}(t)\right\|_{\infty} .
\end{aligned}
$$

Recall that $\nabla Z_{t, s}^{(x, n)}=Z_{t, s}^{(x, n)} \nabla Y_{t, s}^{(x, n)}$. Due to the bounds of the successive derivatives of $r_{n}$ and the semi-martingale decomposition of $\nabla Y_{t, s}^{(x, n)}$, we can prove as in the previous lemma that the map $x \mapsto \nabla Y_{t, s}^{(x, n)}$ is differentiable in $L^{2 p}(\Omega)$. Moreover $\nabla^{2} Y_{t, s}^{(x, n)}$ is computed by differentiating $\nabla Y_{t, s}^{(x, n)}$ under the integral sign. And so is $\nabla_{x} Z, p \geq 1$. We then have:

$$
\nabla^{2} Z_{t, s}^{(x, n)}=Z_{t, s}^{(x, n)} \nabla^{2} Y_{t, s}^{(x, n)}+\nabla Y_{t, s}^{(x, n)} \nabla Z_{t, s}^{(x, n)} .
$$

Integrating by parts, we obtain:

$$
\begin{aligned}
\nabla_{x}^{2} Z_{t, s}^{(x, n)}= & \int_{t}^{s} \nabla^{2} r_{n}\left(r, W_{t, x}^{\sigma}(r)\right)\left(\nabla W_{t, x}^{\sigma}(r)\right)^{2} Z_{t, r}^{(x, n)} d W_{t, 0}(r) \\
& +\int_{t}^{s} \nabla r_{n}\left(r, W_{t, x}^{\sigma}(r)\right) \nabla^{2} W_{t, x}^{\sigma}(r) Z_{t, r}^{(x, n)} d W_{t, 0}(r) \\
& +2 \int_{t}^{s} \nabla r_{n}\left(r, W_{t, x}^{\sigma}(r)\right) \nabla Z_{t, r}^{(x, n)} d W_{t, 0}(r) \\
& +\int_{t}^{s} r_{n}\left(r, W_{t, x}^{\sigma}(r)\right) \nabla_{x}^{2} Z_{t, r}^{(x, n)} d W_{t, 0}(r) .
\end{aligned}
$$

The suitable bounds then yield

$$
E\left|\nabla^{2} Z_{t, s}^{(x, n)}\right|^{2} \leq C \int_{t}^{s}\left\|\nabla^{2} u_{n}(r)\right\|_{\infty}^{2} d r+C,
$$

where the constant $C$ does not depend on $n$.

Repeating the same reasoning involving the rates of increase and the Cauchy-Schwarz inequality as in the previous lemma, we can bound $\nabla^{2} u_{n+1}$ by an expression in terms of $Z_{t}^{(x, n)}, f_{n}, u_{0}, W_{t, x}^{\sigma}$ and their two first derivatives using a first order Taylor expansion. We then obtain

$$
\left|\nabla^{2} u_{n+1}(t, x)\right|^{2} \leq C+C \int_{t}^{T}\left\|\nabla^{2} u_{n}(r)\right\|_{\infty}^{2} d r .
$$

The conclusion of Lemma 3.8 follows from Lemma 3.1. 
Step 3. Existence of a smooth solution

We apply Theorem 15 p.80 in [8] (See Appendix 5.1). Let us define the following sequence of parabolic operators:

$$
\mathcal{L}_{\phi\left(t, x, u_{n}\right)}=\partial_{t}+\phi\left(t, x, u_{n}\right) \cdot \nabla+\frac{1}{2} \Delta_{\sigma}
$$

We can write:

$$
\mathcal{L}_{\phi\left(t, x, u_{n}\right)} u_{n+1}=f_{n} .
$$

Let us verify the assumptions of Friedman's Theorem on any bounded domain $\Xi \subset[0, T) \times \mathbb{R}^{d}$ of the form $\Xi=\left[0, T\left[\times[m, M]\right.\right.$. First we know that $\left(u_{n}\right)$ uniformly converges to a function $u$ on $[0, T] \times \mathbb{R}^{d}$. Second from Steps 1 and 2 we deduce that

$$
\sup _{n \geq 0}\left\|\partial_{t} u_{n}\right\|_{\infty}<\infty
$$

and then

$$
\sup _{n \geq 0}\left(\left\|\partial_{t} u_{n}\right\|_{\infty}+\left\|u_{n}\right\|_{\infty}+\left\|\nabla u_{n}\right\|_{\infty}\right)<\infty
$$

Therefore

$$
\sup _{n \geq 0}\left(\left\|\partial_{t} f_{n}\right\|_{\infty}+\left\|f_{n}\right\|_{\infty}+\left\|\nabla f_{n}\right\|_{\infty}\right)<\infty .
$$

Condition (B) (See Appendix 5.1) i.e. the uniform parabolic condition holds due to Assumption $H_{\Delta}$, whereas Condition (A) holds due to (4.136) and Assumption $H_{\Delta}$. Now the solution $u_{n+1}$ of

$$
\mathcal{L}_{\phi\left(t, x, u_{n}\right)} u_{n+1}=f_{n}
$$

obviously satisfies (4.135). As a consequence, there exists a subsequence of $\left(u_{n+1}\right)$ such that its two first derivatives also converge uniformly on the domain $\Xi$. Moreover $u$ is continuously twice differentiable and verifies

$$
\left(\mathcal{L}_{\phi(t, x, u)} u\right)(t, x)=\left(\partial_{t} u+\phi(t, x, u) \cdot \nabla u+\frac{1}{2} \Delta_{\sigma} u\right)(t, x)=-g(t, x, u(t, x)), \quad(t, x) \in \Xi .
$$

As a conclusion, using Theorem 10 p.72 in [8], we deduce that $u \in C^{\infty}\left([0, T) \times \mathbb{R}^{d}\right)$.

It remains to conclude about the regularity at $T$. By Step 1, we deduce that

$$
\left\|u(t)-u_{0}\right\|_{\infty} \underset{t \rightarrow T}{\longrightarrow} 0 .
$$

Using (3.75) we compute $\delta_{t}\left(u_{n+1}\right)\left(x, y_{\alpha}\right)-\nabla u_{0}(x)$. We bound it by means of Cauchy-Schwarz's inequality, the uniform bound (3.59) and Taylor expansions as in (3.76-3.77). We then let $\alpha \rightarrow 0$ to obtain a bound for $\left\|\nabla u_{n+1}(t)-\nabla u_{0}\right\|_{\infty}$. Using Cauchy-Schwarz's inequality, Inequality (3.71) together with Lemma 3.7, we can let $n \rightarrow \infty$ and hence obtain a bound for $\left\|\nabla u(t)-\nabla u_{0}\right\|_{\infty}$ of the form $C(T-t)$. We finally deduce

$$
\left\|\nabla u(t)-\nabla u_{0}\right\|_{\infty} \underset{t \rightarrow T}{\longrightarrow} 0 .
$$

The same analysis can be performed to show the continuity of the derivatives of higher order. 
Step 4. Unicity of the solution.

Lemma 3.9. The system (4.125) admits at most one bounded solution in $C^{\infty}\left([0, T] \times \mathbb{R}^{d}\right)$.

Proof. Let $u$ and $v$ be two bounded solutions in $C^{\infty}\left([0, T] \times \mathbb{R}^{d}\right)$. We write their implicit FeynmanKac representation; For instance $u$ reads:

$$
u(t, x)=E\left[\int_{t}^{T} f^{u}\left(s, X_{t, x}^{u}(s)\right) d s+u_{0}\left(X_{t, x}^{u}(T)\right)\right],
$$

where

$$
f^{u}(t, x)=g(t, x, u(t, x))
$$

and $X_{t, x}^{u}$ is the stochastic flow defined by

$$
X_{t, x}^{u}(s)=x+\int_{t}^{s} \phi\left(r, X_{t, x}^{u}(r), u\left(r, X_{t, x}^{u}(r)\right)\right) d r+\int_{t}^{s} \sigma\left(r, X_{t, x}^{u}(r)\right) d W_{t, 0}(r), \quad 0 \leq t \leq s \leq T .
$$

Using the same notation and techniques as in Lemma 3.5, we obtain

$$
\gamma(t):=\sup _{x \in \mathbb{R}^{d}}|(u-v)(t, x)|^{2} \leq C E\left|Z_{t, T}^{(x, u)}-Z_{t, T}^{(x, v)}\right|^{2} .
$$

Set $0<T^{\prime} \leq T$. As in Lemma 3.5 we obtain the following inequalities:

$$
\begin{aligned}
E\left|Z_{t, T^{\prime}}^{(x, u)}-Z_{t, T^{\prime}}^{(x, v)}\right|^{2} \leq & C E\left|\int_{t}^{T^{\prime}}(u-v)\left(r, W_{t, x}^{\sigma}(r)\right) Z_{t, r}^{(x, v)} d W_{t, 0}(r)\right|^{2} \\
& +C E\left|\int_{t}^{T^{\prime}} v\left(r, W_{t, x}^{\sigma}(r)\right)\left(Z_{t, r}^{(x, u)}-Z_{t, r}^{(x, v)}\right) d W_{t, 0}(r)\right|^{2} \\
= & C E \int_{t}^{T}\left|(u-v)\left(r, W_{t, x}^{\sigma}(r)\right)\right|^{2}\left|Z_{t, r}^{(x, u)}\right|^{2} d r \\
& +C E \int_{t}^{T^{\prime}} \mid v\left(r,\left.W_{t, x}^{\sigma}(r)\right|^{2}\left|Z_{t, r}^{(x, u)}-Z_{t, r}^{(x, u)}\right|^{2} d r\right. \\
= & C \int_{t}^{T} \gamma(r) d r+C E \int_{t}^{T^{\prime}} E\left|Z_{t, r}^{(x, u)}-Z_{t, r}^{(x, v)}\right|^{2} d r .
\end{aligned}
$$

So due to Gronwall's Lemma (making $T^{\prime}$ vary):

$$
E\left|Z_{t, T^{\prime}}^{(x, u)}-Z_{t, T^{\prime}}^{(x, v)}\right|^{2} \leq C \int_{t}^{T} \gamma(r) d r .
$$

Setting now $T^{\prime}=T$, we thus have:

$$
\gamma(t) \leq C \int_{t}^{T} \gamma(r) d r
$$

Thus $\gamma=0$, which concludes Lemma 3.9. 
Step 5. $L^{2}$ bounds of the solution when $u_{0} \in L^{2}\left(\mathbb{R}^{d}\right)$.

From (3.50-3.24) we obtain:

$$
E\left(Z_{t, T}^{(x, n)}\right)^{2} \leq \exp \left(2 \lambda_{2}(T-t)\right)
$$

Let us assume that $u_{0} \in L^{2}\left(\mathbb{R}^{d}\right)$ and $\|M\|_{L\left([0, T] \times \mathbb{R}^{d}\right)}^{2}<\infty$. From (3.45) and using the Cauchy Schwarz inequality we deduce the following:

$$
\begin{aligned}
\left|u_{n+1}(t, x)\right| & \leq \sqrt{E\left(Z_{t, T}^{(x, n)}\right)^{2}} \sqrt{E\left|\int_{t}^{T} f_{n}\left(s, W_{t, x}^{\sigma}(s)\right) d s+u_{0}\left(W_{t, x}^{\sigma}(T)\right)\right|^{2}} \\
\left|u_{n+1}(t, x)\right|^{2} & \leq 2 E\left(Z_{t, T}^{(x, n)}\right)^{2} E\left((T-t) \int_{t}^{T}\left|f_{n}\left(s, W_{t, x}^{\sigma}(s)\right)\right|^{2} d s+\left|u_{0}\left(W_{t, x}^{\sigma}(T)\right)\right|^{2}\right)
\end{aligned}
$$

By Th. 4.6 .5 p.173 in [11], we know that $x \mapsto W_{t, x}^{\sigma}(s)$ is a $C^{1}$ diffeomorphism. Let $J_{t, x}^{\sigma}(s)$ be the module of the Jacobian of its inverse. Using $e^{|z|} \leq e^{z}+e^{-z}$, the martingale property of the Doleans exponential, and the bounds of the successive derivatives of $\sigma$, we can prove that $\left\|E J^{\sigma}\right\|_{\infty}<\infty$. We then obtain by integrating in $x$ the last inequality:

$$
\begin{aligned}
\left\|u_{n+1}(t)\right\|_{2}^{2} & \leq 2 E\left(Z_{t, T}^{(x, n)}\right)^{2}\left\|E J^{\sigma}\right\|_{\infty}\left((T-t) \int_{t}^{T} \int_{\mathbb{R}^{d}}\left|f_{n}(s, x)\right|^{2} d x d s+\int_{\mathbb{R}^{d}}\left|u_{0}(x)\right|^{2} d x\right) \\
& \leq 2\left\|E J^{\sigma}\right\|_{\infty} e^{2 \lambda_{2}(T-t)}\left(T \int_{t}^{T}\left\|f_{n}(s)\right\|_{2}^{2} d s+\left\|u_{0}\right\|_{2}^{2}\right) \\
& \leq 4\left\|E J^{\sigma}\right\|_{\infty} e^{2 \lambda_{2}(T-t)}\left(T \int_{t}^{T}\|M(s)\|_{2}^{2} d s+c_{1}^{2} T \int_{t}^{T}\left\|u_{n}(s)\right\|_{2}^{2} d s+\left\|u_{0}\right\|_{2}^{2}\right) .
\end{aligned}
$$

Setting $\lambda_{3}=4\left\|E J^{\sigma}\right\|_{\infty}^{2} e^{2 \lambda_{2} T}$, we now apply Lemma 3.1 with $k_{0}=\widetilde{K}_{0}=\lambda_{3}\left\|u_{0}\right\|_{2}^{2}, K_{1}=\lambda_{3} c_{1}^{2} T$ and $\theta(s)=\lambda_{3}\|M(s)\|_{2}^{2}$ and we get:

$$
\left\|u_{n+1}(t)\right\|_{2}^{2} \leq\left(\lambda_{3}\left\|u_{0}\right\|_{2}^{2}+\int_{0}^{T} \lambda_{3}\|M(s)\|_{2}^{2} d s\right) e^{\lambda_{3} c_{1}^{2} T(T-t)}-\int_{0}^{t} \lambda_{3}\|M(s)\|_{2}^{2} d s .
$$

This concludes the Theorem.

3.3. Source term of the form $u^{\alpha}$. We consider the following Cauchy problem

$$
\left\{\begin{aligned}
\partial_{t} v(t, x)+(\phi(v) \cdot \nabla) v(t, x)-\nu \Delta v(t, x) & =\beta v^{\alpha}(t, x) \\
v(0, x) & =v_{0}(x),
\end{aligned}\right.
$$

where $\nu>0, \alpha \geq 2, \phi: \mathbb{R}^{d} \rightarrow \mathbb{R}^{d}, v_{0}: \mathbb{R}^{d} \rightarrow \mathbb{R}^{d}$ and $v:[0, T] \times \mathbb{R}^{d} \rightarrow \mathbb{R}^{d}$ is the unknown. By $x^{\alpha}$, we mean $\left(x^{1 \alpha}, \cdots, x^{d \alpha}\right)$.

Theorem 3.10. Let $T>0, \alpha \geq 2$, $\phi$ be a function in $C^{\infty}\left(\mathbb{R}^{d}\right)$ whose first and second derivatives are bounded, $v_{0} \in C^{\infty}\left(\mathbb{R}^{d}\right)$ with bounded derivatives of all orders and assume that

$$
\beta\left\|v_{0}\right\|_{\infty}^{\alpha-2} e^{\left\|v_{0}\right\|_{\infty} T(\alpha-1)} \leq 1
$$

Then there exists a unique solution $v$ in $C^{\infty}$ verifying the Cauchy problem (3.98). 


\section{Remark 3.}

(i) We consider the case where $\phi$ is in $C^{\infty}\left(\mathbb{R}^{d}\right)$ with first and second derivatives bounded, only for simplicity.

(ii) In the case where $v_{0} \in C^{\infty}\left(\mathbb{R}^{d}\right) \cap L^{2}\left(\mathbb{R}^{d}\right)$, this theorem is actually a particular case of well known results on local existence for quasi-linear PDEs (see [18] e.g.).

Proof. The proof of this theorem is very similar to that of Theorem 3.4. Using the change of variable $u(t, x)=v(T-t,-x)$, we turn the initial Cauchy system into the following backward one:

$$
\left\{\begin{aligned}
\partial_{t} u(t, x)+(\phi(u) \cdot \nabla) u(t, x)+\frac{1}{2} \sigma^{2} \Delta u(t, x) & =-\beta u^{\alpha}(t, x) \\
u(T, x) & =u_{0}(x),
\end{aligned}\right.
$$

where $u_{0}:=v_{0}$ and $\sigma=\sqrt{2 \nu}$.

Let us define by induction the sequence $u_{n}:[0, T] \times \mathbb{R}^{d} \rightarrow \mathbb{R}^{d}, n \geq 0$ as:

$$
\begin{aligned}
u_{0}(t, x) & =u_{0}(x) \\
u_{n+1}(t, x) & =E\left[\int_{t}^{T} f_{n}\left(s, X_{t, x}^{(n)}(s)\right) d s+u_{0}\left(X_{t, x}^{(n)}(T)\right)\right],
\end{aligned}
$$

where

$$
f_{n}(t, x)=\beta u_{n}^{\alpha}(t, x),
$$

and $X_{t, x}^{(n)}$ is the stochastic flow defined by:

$$
X_{t, x}^{(n)}(s)=x+\int_{t}^{s} w_{n}\left(r, X_{t, x}^{(n)}(r)\right) d r+W_{t, 0}^{\sigma}(s), \quad 0 \leq t \leq s \leq T,
$$

with

$$
w_{n}(t, x)=\phi\left(u_{n}(t, x)\right) .
$$

Because of the Feynman-Kac representation of $u_{n+1}$, we get

$$
\left\|u_{n+1}\right\|_{\infty} \leq \beta \int_{t}^{T}\left\|u_{n}(s)\right\|_{\infty}^{\alpha} d s+\left\|u_{0}\right\|_{\infty} .
$$

By induction, we assume that

$$
\left\|u_{n}(s)\right\|_{\infty} \leq \sum_{k=0}^{n} \frac{\left\|u_{0}\right\|_{\infty}^{k+1}(T-s)^{k}}{k !}, \quad s \in[0,1] .
$$

Then

$$
\left\|u_{n}(s)\right\|_{\infty} \leq\left\|u_{0}\right\|_{\infty} e^{\left\|u_{0}\right\|_{\infty} T}
$$

and

$$
\begin{aligned}
\left\|u_{n+1}\right\|_{\infty} & \leq \beta\left\|u_{0}\right\|_{\infty}^{\alpha-1} e^{\left\|u_{0}\right\|_{\infty} T(\alpha-1)} \int_{t}^{T}\left\|u_{n}(s)\right\|_{\infty} d s+\left\|u_{0}\right\|_{\infty} \\
& \leq\left\|u_{0}\right\|_{\infty} \sum_{k=0}^{n} \frac{\left\|u_{0}\right\|_{\infty}^{k+1}(T-s)^{k+1}}{(k+1) !}+\left\|u_{0}\right\|_{\infty} \\
& \leq \sum_{k=0}^{n+1} \frac{\left\|u_{0}\right\|_{\infty}^{k+1}(T-s)^{k}}{k !} .
\end{aligned}
$$


We can then conclude that $\sup _{n, t}\left\|u_{n}(t)\right\|_{\infty}<\infty$ and $\sup _{n, t}\left\|f_{n}(t)\right\|_{\infty}<\infty$.

The sequel is similar; To show that $\left(u_{n}\right)_{n}$ is uniformly convergent, we have to estimate

$$
\left(f_{n+1}-f_{n}\right)\left(s, x+\sigma W_{t, 0}(s)\right)=\beta\left(u_{n+1}^{\alpha}-u_{n}^{\alpha}\right)\left(s, x+\sigma W_{t, 0}(s)\right) .
$$

But the Taylor expansion component wise yields the equality $\left(X^{i}\right)^{\alpha}-\left(Y^{i}\right)^{\alpha}=\alpha\left(\theta^{i}\right)^{\alpha-1}\left(X^{i}-Y^{i}\right)$ where $\theta^{i} \in\left[X^{i}, Y^{i}\right]$. In our case, $\theta^{i} \in\left[u_{n+1}^{i}, u_{n}^{i}\right]$ is bounded so that

$$
\left|\left(f_{n+1}-f_{n}\right)\left(s, x+\sigma W_{t, 0}(s)\right)\right| \leq c\left|\left(u_{n+1}-u_{n}\right)\left(s, x+\sigma W_{t, 0}(s)\right)\right|
$$

and we can conclude as in the proof of Theorem 3.4.

As for the estimation of $\left\|\nabla u_{n}\right\|_{\infty}$, we have

$$
\left|\nabla f_{n}(t, x)\right|=\beta\left|\alpha \nabla u_{n}(t, x) u_{n}^{\alpha-1}(t, x)\right| \leq c|| \nabla u_{n}(t, x) \|_{\infty} .
$$

Thus, we can conclude as in the proof of Theorem 3.4 that $\sup _{n}\left\|\nabla u_{n}\right\|_{\infty}<\infty$. Finally,

$$
\nabla^{2} f_{n}(t, x)=\beta \alpha(\alpha-1)\left(\nabla u_{n}(t, x)\right)^{2} u_{n}^{\alpha-2}(t, x)+\beta \alpha \nabla^{2} u_{n}(t, x) u_{n}^{\alpha-1}(t, x) .
$$

It follows that

$$
\left|\nabla^{2} f_{n}(t, x)\right| \leq c\left|\nabla^{2} u_{n}(t, x)\right|+c
$$

and we can also conclude in the same way that $\sup _{n}\left\|\nabla^{2} u_{n}\right\|_{\infty}<\infty$.

Therefore, it suffices to conclude by using Theorem 15 p.80 in [8].

The uniqueness is obtained by the same reasoning: setting $\gamma(t):=\sup _{x \in \mathbb{R}^{d}}|(u-v)(t, x)|^{2}$, we deduce

$$
\gamma(t) \leq C \int_{t}^{T} \gamma(r) d r
$$

since $\alpha \geq 2$ implies $\left(u^{i}\right)^{\alpha}(t, x)-\left(v^{i}\right)^{\alpha}(t, x)=\left(u^{i}(t, x)-v^{i}(t, x)\right) l^{i}(t, x)$ where $l^{i}$ are bounded functions.

\section{Hyperbolic CASE}

This section aims at studying the following hyperbolic PDE:

$$
\left\{\begin{aligned}
\partial_{t} v(t, x)+(\phi(t, x, v) \cdot \nabla) v(t, x) & =h(t, x, v(t, x)) \\
v(0, x) & =v_{0}(x),
\end{aligned}\right.
$$

where $\phi: \mathbb{R}^{d} \rightarrow \mathbb{R}^{d}, \nu:[0, T] \times \mathbb{R}^{d} \rightarrow \mathbb{R}^{d} \otimes \mathbb{R}^{d}, h:[0, T] \times \mathbb{R}^{d} \times \mathbb{R}^{d} \rightarrow \mathbb{R}^{d}, v_{0}: \mathbb{R}^{d} \rightarrow \mathbb{R}^{d}$ are given while $v:[0, T] \times \mathbb{R}^{d} \rightarrow \mathbb{R}^{d}$ is the unknown.

Recall that for all $t \in[0, T]$

$$
\lambda_{1}(t):=\left(\left\|v_{0}\right\|_{\infty}+\int_{0}^{T}\|M(s)\|_{\infty} d s\right) e^{c_{1} t}-\int_{t}^{T}\|M(s)\|_{\infty} d s .
$$

We then define the following constants:

$$
\begin{aligned}
\lambda_{1} & :=\lambda_{1}(T) \\
\beta_{g, \phi, v_{0}} & :=\left\|\nabla v_{0}\right\|_{\infty} \Phi_{2}^{1}\left(\lambda_{1}\right)\left(e^{-\Phi_{1}^{1}\left(\lambda_{1}\right) T} \frac{\left\|\nabla_{x} g\right\|_{\infty}}{2\left\|\nabla v_{0}\right\|_{\infty}}+\left\|\nabla_{y} g\right\|_{\infty}\right)^{-1} .
\end{aligned}
$$

The main result of this section is the following theorem. 
Theorem 4.1. Let $T>0, \phi$ be a function in $C^{\infty}\left(\mathbb{R}^{d}\right)$ whose first and second derivatives are bounded, $v_{0} \in C_{b, 4}^{4}\left(\mathbb{R}^{d}\right), h:[0, T] \times \mathbb{R}^{d} \times \mathbb{R}^{d} \rightarrow \mathbb{R}^{d}$ be a $C^{\infty}$ function satisfying Assumption $(H)$.

Then there exists $T^{*} \in(0, T]$ and a unique bounded solution $v$ in $C^{2}\left(\left[0, T^{*}\right] \times \mathbb{R}^{d}\right)$ verifying the Cauchy problem (4.106). Moreover:

$$
\sup _{t \in\left[0, T^{*}\right]}\|v(t)\| \leq \lambda_{1}
$$

and the following lower bound for $T^{*}$ holds:

$$
T^{*} \geq \frac{e^{-\Phi_{1}^{1}\left(\lambda_{1}\right) T}}{2 \Phi_{2}^{1}\left(\lambda_{1}\right)\left\|\nabla v_{0}\right\|_{\infty}} \log \left(\frac{1+2 \beta_{g, \phi, v_{0}}}{1+\beta_{g, \phi, v_{0}}}\right) .
$$

Proof. The proof is based on the following steps. First, based on the associated backward Cauchy system we define and study a collection of sequence $\sigma \mapsto u^{\sigma}:=\left(u_{n}^{\sigma}\right)$ derived from a FeynmanKac representation where the coefficient diffusion sequence $\sigma=\left(\sigma_{n}(\cdot)\right)_{n}$ lie in the family $\Sigma$ of all sequences such that for all $n \in \mathbb{N}, \sigma_{n}(\cdot) \in C_{b}^{\infty}([0, T],(0, \infty))$. In Step 1, we obtain uniform bounds of the derivatives of $\left(u_{n}^{\sigma}\right)$ in $n$ and $\sigma$ on a small interval. In Step 2, we show the uniform convergence of a particular sequence $u^{*}:=\left(u_{n}^{\sigma^{*}}\right)$ for a suitable sequence $\sigma^{*}$ converging to 0 as $n \rightarrow \infty$. In Step 3, we conclude that the limit $u^{*}$ of $\left(u_{n}^{\sigma^{*}}\right)$ is a smooth solution of the Cauchy system by using the previous results and applying a Friedman's Theorem (Th. 15 p.80 in [8]) on uniform parabolic PDEs. In Step 4, we prove the uniqueness of the solution and eventually in Step 5, we obtain suitable bounds in $L^{\infty}$ and $L^{2}$ for this solution.

Using the change of variable $u(t, x)=v(T-t,-x)$, we turn the initial Cauchy system into the following backward one:

$$
\left\{\begin{aligned}
\partial_{t} u(t, x)+(\phi(t, x, u) \cdot \nabla) u(t, x) & =-g(t, x, u(t, x)) \\
u(T, x) & =u_{0}(x),
\end{aligned}\right.
$$

where $u_{0}:=v_{0}$ and $g(t, x, y)=h(T-t,-x, y)$. Note that $g$ satisfies the same conditions than $h$.

For a given sequence $\sigma=\left(\sigma_{n}(t)\right)_{n} \in \Sigma$, let us define by induction the sequence $u^{\sigma}=\left(u_{n}^{\sigma}\right)$ : $[0, T] \times \mathbb{R}^{d} \rightarrow \mathbb{R}^{d}, n \geq 0$ as:

$$
\begin{aligned}
u_{0}^{\sigma}(t, x) & =u_{0}(x) \\
u_{n+1}^{\sigma}(t, x) & =E\left[\int_{t}^{T} f_{n}^{\sigma}\left(s, X_{t, x}^{(n)}(s)\right) d s+u_{0}\left(X_{t, x}^{(n)}(T)\right)\right],
\end{aligned}
$$

where

$$
f_{n}(t, x)=g\left(t, x, u_{n}^{\sigma}(t, x)\right)
$$

and $X_{t, x}^{(n)}$ is the stochastic flow defined by:

$$
X_{t, x}^{(n)}(s)=x+\int_{t}^{s} w_{n}^{\sigma}\left(r, X_{t, x}^{(n)}(r)\right) d r+\int_{t}^{s} \sigma_{n}(r) d W_{t, 0}(r), \quad 0 \leq t \leq s \leq T,
$$

with

$$
w_{n}^{\sigma}(t, x)=\phi\left(t, x, u_{n}^{\sigma}(t, x)\right) .
$$


Note that $u_{n+1}^{\sigma}$ is derived from the Feynman-Kac representation as the solution of the following PDE:

$$
\left\{\begin{aligned}
\partial_{t} u_{n+1}^{\sigma}(t, x)+\left(w_{n}^{\sigma} \cdot \nabla\right) u_{n+1}^{\sigma}(t, x)+\frac{1}{2} \sigma_{n}^{2}(t) \Delta u_{n+1}^{\sigma}(t, x) & =f_{n}^{\sigma}(t, x) \\
u_{n+1}^{\sigma}(T, x) & =u_{0}(x) .
\end{aligned}\right.
$$

From Theorem 10 p. 72 in [8], we deduce by induction that $u_{n+1}^{\sigma} \in C^{\infty}\left([0, T] \times \mathbb{R}^{d}\right)$.

Lemma 4.2. There exists $T_{0} \in[0, T)$ and a constant $C_{0}^{*}$ such that for all $t \in\left[T_{0}, T\right]$ and all $\sigma \in \Sigma$.

$$
\sup _{n \in \mathbb{N}}\left(\left\|u_{n}^{\sigma}(t)\right\|_{\infty}+\left\|\nabla u_{n}^{\sigma}(t)\right\|_{\infty}+\cdots+\left\|\nabla^{4} u_{n}^{\sigma}(t)\right\|_{\infty}\right) \leq C_{0}^{*} .
$$

Proof. As in Step 1 of Theorem 3.4, we deduce that for all $n \geq 0$ and all $\sigma \in \Sigma,\left\|u_{n}^{\sigma}\right\|_{\infty} \leq \lambda_{1}$.

From (4.116), we compute

$$
\nabla X_{t, x}^{(n)}(s)=\mathrm{Id}+\int_{t}^{s} \nabla w_{n}^{\sigma}\left(r, X_{t, x}^{(n)}(r)\right) \nabla X_{t, x}^{(n)}(r) d r, \quad 0 \leq t \leq s \leq T .
$$

From (3.25) we have $\left\|\nabla w_{n}^{\sigma}(r)\right\|_{\infty} \leq \Phi_{1}^{1}\left(\lambda_{1}\right)+\Phi_{2}^{1}\left(\lambda_{1}\right)\left\|\nabla u_{n}^{\sigma}(r)\right\|_{\infty}$, and then

$$
\left|\nabla X_{t, x}^{(n)}(s)\right| \leq e^{\Phi_{1}^{1}\left(\lambda_{1}\right) T} \exp \left(\Phi_{2}^{1}\left(\lambda_{1}\right) \int_{t}^{s}\left\|\nabla u_{n}^{\sigma}(r)\right\|_{\infty} d r\right) .
$$

Computing

$$
\begin{aligned}
\nabla u_{n+1}^{\sigma}(t, x)=E & {\left[\int_{t}^{T} \nabla_{x} g\left(s, X_{t, x}^{(n)}(s), u_{n}^{\sigma}\left(r, X_{t, x}^{(n)}(s)\right)\right) \nabla X_{t, x}^{(n)}(s) d s\right.} \\
& +\int_{t}^{T} \nabla_{y} g\left(s, X_{t, x}^{(n)}(s), u_{n}^{\sigma}\left(r, X_{t, x}^{(n)}(s)\right)\right) \nabla u_{n}^{\sigma}\left(t, X_{t, x}^{(n)}(s)\right) \nabla X_{t, x}^{(n)}(s) d s \\
& \left.+\nabla u_{0}\left(X_{t, x}^{(n)}(T) \nabla X_{t, x}^{(n)}(T)\right)\right],
\end{aligned}
$$

we thus deduce

$$
\begin{aligned}
e^{-\Phi_{1}^{1}\left(\lambda_{1}\right) T}\left|\nabla u_{n+1}^{\sigma}(t, x)\right| \leq \| & \nabla_{x} g \|_{\infty} \int_{t}^{T} \exp \left(\Phi_{2}^{1}\left(\lambda_{1}\right) \int_{t}^{s}\left\|\nabla u_{n}^{\sigma}(r)\right\|_{\infty} d r\right) d s \\
& +\left\|\nabla_{y} g\right\|_{\infty} \int_{t}^{T}\left\|\nabla u_{n}^{\sigma}(s)\right\|_{\infty} \exp \left(\Phi_{2}^{1}\left(\lambda_{1}\right) \int_{t}^{s}\left\|\nabla u_{n}^{\sigma}(r)\right\|_{\infty} d r\right) d s \\
& +\left\|\nabla u_{0}\right\|_{\infty} \exp \left(\Phi_{2}^{1}\left(\lambda_{1}\right) \int_{t}^{T}\left\|\nabla u_{n}^{\sigma}(r)\right\|_{\infty} d r\right)
\end{aligned}
$$

Let us define the constant

$$
C_{1}=2 e^{\Phi_{1}^{1}\left(\lambda_{1}\right) T}\left\|\nabla u_{0}\right\|_{\infty}
$$

and the decreasing function $\eta_{C_{1}}$ on $[0, T]$ by

$$
\eta_{C_{1}}(t):=e^{\Phi_{1}^{1}\left(\lambda_{1}\right) T}\left(e^{\Phi_{2}^{1}\left(\lambda_{1}\right) C_{1}(T-t)}\left(C_{g, \phi, \lambda_{1}}+\left\|\nabla u_{0}\right\|_{\infty}\right)-C_{g, \phi, \lambda_{1}}\right)
$$

where

$$
C_{g, \phi, \lambda_{1}}:=\frac{\left\|\nabla_{x} g\right\|_{\infty}}{\Phi_{2}^{1}\left(\lambda_{1}\right) C_{1}}+\frac{\left\|\nabla_{y} g\right\|_{\infty}}{\Phi_{2}^{1}\left(\lambda_{1}\right)}
$$


Let us choose $T_{0}<T$ such that $\eta_{C_{1}}\left(T_{0}\right)=C_{1}$, that is

$$
T_{0}:=T-\frac{1}{\Phi_{2}^{1}\left(\lambda_{1}\right) C_{1}} \log \left(\frac{C_{g, \phi, \lambda_{1}}+2\left\|\nabla u_{0}\right\|_{\infty}}{C_{g, \phi, \lambda_{1}}+\left\|\nabla u_{0}\right\|_{\infty}}\right) .
$$

Let us prove by induction that

$$
\sup _{n \in \mathbb{N}, t \in\left[T_{0}, T\right]}\left\|\nabla u_{n}(t)\right\|_{\infty} \leq C_{1} .
$$

So assume for some $n$ that for all $t \in\left[T_{0}, T\right],\left\|\nabla u_{n}(t)\right\|_{\infty} \leq C_{1}$. Hence,

$$
\begin{aligned}
e^{-\Phi_{1}^{1}\left(\lambda_{1}\right) T}\left|\nabla u_{n+1}^{\sigma}(t, x)\right| \leq \| & \nabla_{x} g \|_{\infty} \int_{t}^{T} \exp \left(\Phi_{2}^{1}\left(\lambda_{1}\right) C_{1}(s-t)\right) d s \\
& +\left\|\nabla_{y} g\right\|_{\infty} \int_{t}^{T}\left\|\nabla u_{n}^{\sigma}(s)\right\| \exp \left(\Phi_{2}^{1}\left(\lambda_{1}\right) C_{1}(s-t)\right) d s \\
& +\left\|\nabla u_{0}\right\|_{\infty} \exp \left(\Phi_{2}^{1}\left(\lambda_{1}\right) C_{1}(T-t)\right),
\end{aligned}
$$

and

$$
\begin{aligned}
e^{-\Phi_{1}^{1}\left(\lambda_{1}\right) T}\left|\nabla u_{n+1}^{\sigma}(t, x)\right| \leq \frac{\left\|\nabla_{x} g\right\|_{\infty}}{\Phi_{2}^{1}\left(\lambda_{1}\right) C_{1}}\left(e^{\Phi_{2}^{1}\left(\lambda_{1}\right) C_{1}(T-t)}-1\right) \\
+\frac{\left\|\nabla_{y} g\right\|_{\infty}}{\Phi_{2}^{1}\left(\lambda_{1}\right)}\left(e^{\Phi_{2}^{1}\left(\lambda_{1}\right) C_{1}(T-t)}-1\right) \\
+\left\|\nabla u_{0}\right\| \exp \left(\Phi_{2}^{1}\left(\lambda_{1}\right) C_{1}(T-t)\right) .
\end{aligned}
$$

Therefore

$$
\left|\nabla u_{n+1}^{\sigma}(t, x)\right| \leq \eta_{C_{1}}(t) \leq \eta_{C_{1}}\left(T_{0}\right)=C_{1}, \quad T_{0} \leq t \leq T,
$$

which proves (4.124).

Let us now prove that $\left|\nabla^{2} X_{t, x}^{(n)}(s)\right| \leq C+C \int_{t}^{s}\left\|\nabla^{2} u_{n}^{\sigma}(r)\right\|_{\infty} d r$, where $C$ is a constant independent of $n$ and $\sigma$. First write:

$$
\nabla^{2} X_{t, x}^{(n)}(s)=\int_{t}^{s} \nabla^{2} w_{n}^{\sigma}\left(r, X_{t, x}^{(n)}(r)\right) \nabla X_{t, x}^{(n)}(r) d r+\int_{t}^{s} \nabla w_{n}^{\sigma}\left(r, X_{t, x}^{(n)}(r)\right) \nabla^{2} X_{t, x}^{(n)}(r) d r .
$$

From (3.24-3.25) and the uniform bound (4.124) we obtain for all $n \in \mathbb{N}$ and all $\sigma \in \Sigma$

$$
\begin{aligned}
\left\|\nabla w_{n}(r)\right\|_{\infty} & \leq C \\
\left\|\nabla^{2} w_{n}(r)\right\|_{\infty} & \leq C+C\left\|\nabla^{2} u_{n}^{\sigma}(r)\right\|_{\infty} .
\end{aligned}
$$

(Recall that $C$ can change from line to line) Moreover from (4.120-4.124), we deduce that

$$
\sup _{x, n, T_{0} \leq t \leq s \leq T}\left|\nabla X_{t, x}^{(n)}(s)\right|<\infty .
$$

Thus

$$
\left|\nabla^{2} X_{t, x}^{(n)}(s)\right| \leq C+C \int_{t}^{s}\left\|\nabla^{2} u_{n}^{\sigma}(r)\right\|_{\infty} d r+C \int_{t}^{s}\left|\nabla^{2} X_{t, x}^{(n)}(r)\right| d r .
$$

Therefore by Gronwall's lemma

$$
\left|\nabla^{2} X_{t, x}^{(n)}(s)\right| \leq C+C \int_{t}^{s}\left\|\nabla^{2} u_{n}^{\sigma}(r)\right\|_{\infty} d r
$$


We then compute $\nabla^{2} u_{n+1}$ using (4.121), and we deduce from the last inequality

$$
\left\|\nabla^{2} u_{n+1}^{\sigma}(t)\right\|_{\infty} \leq C+C \int_{t}^{T}\left\|\nabla^{2} u_{n}^{\sigma}(r)\right\|_{\infty} d r .
$$

So we finally obtain a constant $C_{2}$ such for all $\sigma$ and $n$

$$
\left\|\nabla^{2} u_{n+1}^{\sigma}(t)\right\|_{\infty} \leq C_{2}, \quad t \in\left[T_{0}, T\right] .
$$

Using similar reasonings, we obtain uniform bounds $C_{3}$ and $C_{4}$ in $n, \sigma \in \Sigma$ and $t \in\left[T_{0}, T\right]$ for $\left\|\nabla^{3} u_{n+1}^{\sigma}\right\|_{\infty}$ and $\left\|\nabla^{4} u_{n+1}^{\sigma}\right\|_{\infty}$. Setting $C_{1}^{*}=\max \left\{\lambda_{1}, C_{j}, 1 \leq j \leq 4\right\}$, we conclude the Lemma.

Let us now prove the following

Lemma 4.3. There exists a constant $C_{1}^{*}$ such that for all $t \in\left[T_{0}, T\right]$, all $\sigma \in \Sigma$ and all $n \geq 0$

$$
\left\|\left(u_{n+1}^{\sigma}-u_{n}^{\sigma}\right)(t)\right\|_{\infty} \leq C_{1}^{*} \int_{t}^{T}\left\|\left(u_{n}^{\sigma}-u_{n-1}^{\sigma}\right)(r)\right\|_{\infty} d r+C_{1}^{*} \sqrt{\int_{t}^{T}\left(\sigma_{n-1}(r)-\sigma_{n}(r)\right)^{2} d r}
$$

Proof. We write

$$
\begin{aligned}
\left(u_{n+1}^{\sigma}-u_{n}^{\sigma}\right)(t, x)= & E \int_{t}^{T}\left(g\left(s, X_{t, x}^{(n)}(s), u_{n}^{\sigma}\left(s, X_{t, x}^{(n)}(s)\right)\right)-g\left(s, X_{t, x}^{(n-1)}(s), u_{n}^{\sigma}\left(s, X_{t, x}^{(n)}(s)\right)\right)\right. \\
& +g\left(s, X_{t, x}^{(n-1)}(s), u_{n}^{\sigma}\left(s, X_{t, x}^{(n)}(s)\right)\right)-g\left(s, X_{t, x}^{(n-1)}(s), u_{n}^{\sigma}\left(s, X_{t, x}^{(n-1)}(s)\right)\right) \\
& \left.+g\left(s, X_{t, x}^{(n-1)}(s), u_{n}^{\sigma}\left(s, X_{t, x}^{(n-1)}(s)\right)\right)-g\left(s, X_{t, x}^{(n-1)}(s), u_{n-1}^{\sigma}\left(s, X_{t, x}^{(n-1)}(s)\right)\right)\right) d s \\
& +E\left[u_{0}\left(X_{t, x}^{(n)}(T)\right)-u_{0}\left(X_{t, x}^{(n-1)}(T)\right)\right] .
\end{aligned}
$$

Due to the uniform bounds of $\nabla_{x} g, \nabla_{y} g \nabla u_{n}^{\sigma}, \nabla_{y} g$ and $\nabla u_{0}$ in $n$ and $\sigma \in \Sigma$, we deduce a constant $d_{0}$ independent of $\sigma$ and $n$ such that

$$
\begin{aligned}
\left|u_{n+1}^{\sigma}-u_{n}^{\sigma}\right|(t, x) \leq & d_{0} \int_{t}^{T} E\left|X_{t, x}^{(n)}(s)-X_{t, x}^{(n-1)}(s)\right| d s \\
& +d_{0} \int_{t}^{T}\left\|u_{n}^{\sigma}(s)-u_{n-1}^{\sigma}(s)\right\|_{\infty} d s \\
& +E\left|X_{t, x}^{(n)}(T)-X_{t, x}^{(n-1)}(T)\right|
\end{aligned}
$$

But

$$
\begin{aligned}
X_{t, x}^{(n)}(s)-X_{t, x}^{(n-1)}(s)= & \int_{t}^{s}\left(w_{n}\left(r, X_{t, x}^{(n)}(r)\right)-w_{n-1}\left(r, X_{t, x}^{(n)}(r)\right)\right) d r \\
& +\int_{t}^{s}\left(w_{n-1}\left(r, X_{t, x}^{(n)}(r)\right)-w_{n-1}\left(r, X_{t, x}^{(n-1)}(r)\right)\right) d r \\
& +\int_{t}^{T}\left(\sigma_{n-1}(r)-\sigma_{n}(r)\right) d W_{t, 0}(r) .
\end{aligned}
$$


Therefore, due to the uniform bounds of $\nabla \phi$ and $\nabla u_{n}^{\sigma}$, and Ito's isometry, we deduce a constant $d_{1}$ independent of $\sigma$ and $n$ such that

$$
\begin{aligned}
E\left|X_{t, x}^{(n)}(s)-X_{t, x}^{(n-1)}(s)\right| \leq & d_{1} \int_{t}^{T}\left\|u_{n}^{\sigma}(r)-u_{n-1}^{\sigma}(r)\right\|_{\infty} d r \\
& +d_{1} \int_{t}^{s} E\left|X_{t, x}^{(n)}(s)-X_{t, x}^{(n-1)}(r)\right| d r \\
& +\sqrt{\int_{t}^{T}\left(\sigma_{n-1}(r)-\sigma_{n}(r)\right)^{2} d r}
\end{aligned}
$$

We use Gronwall's Lemma to obtain a constant $d_{2}$ independent of $\sigma$ and $n$ such that

$$
\begin{aligned}
E\left|X_{t, x}^{(n)}(s)-X_{t, x}^{(n-1)}(s)\right| \leq & d_{2} \int_{t}^{T}\left\|u_{n}^{\sigma}(r)-u_{n-1}^{\sigma}(r)\right\|_{\infty} d r \\
& +d_{2} \sqrt{\int_{t}^{T}\left(\sigma_{n-1}(r)-\sigma_{n}(r)\right)^{2} d r}
\end{aligned}
$$

Using (4.125), we finally reach the conclusion.

Let us fix $\widetilde{T}>T$ and $p_{0} \in \mathbb{N}^{*}$ such that

$$
2 \sqrt{\frac{\widetilde{T}}{p_{0}+1}} \leq 1 / 2
$$

Set $C_{*}=\max \left(C_{0}^{*}, C_{1}^{*}, \frac{p_{0} !^{\frac{1}{!^{\left(p_{0}-1\right)}}}}{(\widetilde{T}-T)^{\frac{p_{0}}{2\left(p_{0}-1\right)}}}\right)$. In particular, we have:

$$
\frac{C_{*}{ }^{p_{0}}(\widetilde{T}-T)^{p_{0} / 2}}{\sqrt{p_{0} !}} \geq C_{*} .
$$

Note that Lemma 4.3 holds if we replace $C_{1}^{*}$ by $C_{*}$. Finally, let us define for all $t \in[0, \widetilde{T}]$

$$
\sigma_{n}^{*}(t):=\frac{C_{*}^{n+p_{0}}(\widetilde{T}-t)^{\frac{n+p_{0}}{2}}}{\sqrt{\left(n+p_{0}+1\right) !}}, \quad n \geq 1 .
$$

Lemma 4.4. The sequence $u=u^{\sigma^{*}}=\left(u_{n}\right)$ uniformly converges on $\left[T_{0}, T\right] \times \mathbb{R}^{d}$.

Proof. Since

$$
0 \leq \frac{\sigma_{n+1}^{*}}{\sigma_{n}^{*}}(t) \leq \frac{C_{*} \widetilde{T}^{\frac{1}{2}}}{\sqrt{\left(n+p_{0}+2\right)}} \underset{n \rightarrow \infty}{\longrightarrow} 0,
$$

there exists $n_{0}$ such that $\left(\sigma_{n}^{*}\right)_{n \geq n_{0}}$ is decreasing uniformly on $\left[T_{0}, T\right]$ and we choose $n_{0}$ sufficiently large so that

$$
\frac{C_{*}^{n_{0}}(\widetilde{T}-t)^{\frac{n_{0}}{2}}}{\sqrt{n_{0} !}} \sqrt{\left(p_{0}+1\right) !} \leq 1
$$


Morevover, $t \mapsto \sigma_{n}^{*}(t)$ is decreasing $\left[T_{0}, T\right]$ for all $n \in \mathbb{N}$. Then

$$
\begin{aligned}
\sqrt{\int_{t}^{T}\left(\sigma_{n}^{*}(r)-\sigma_{n+1}^{*}(r)\right)^{2} d r} & \leq \sqrt{\int_{t}^{T}\left(\sigma_{n}^{*}(r)\right)^{2} d r} \\
& \leq \sigma_{n}^{*}(t) \sqrt{\widetilde{T}-t} .
\end{aligned}
$$

Consider the sequence $\left(\alpha_{n}\right)$ defined for all $n \in \mathbb{N}$ as

$$
\alpha_{n}(t):= \begin{cases}\left\|\left(u_{n+1+n_{0}}-u_{n+n_{0}}\right)(t)\right\|_{\infty}, & t \in[0, T] \\ 0, & t \in[T, \widetilde{T}] .\end{cases}
$$

Let us prove by induction that for all $n$ and all $t \in\left[T_{0}, \widetilde{T}\right]$

$$
\alpha_{n}(t) \leq 2 \frac{C_{*}^{p_{0}+n}(\widetilde{T}-t)^{\left(p_{0}+n\right) / 2}}{\sqrt{\left(p_{0}+n\right) !}} .
$$

We can easily check that for all $t \in\left[T_{0}, \widetilde{T}\right]$,

$$
\alpha_{0}(t) \leq 2 \frac{C_{*}^{p_{0}}(\widetilde{T}-t)^{p_{0} / 2}}{\sqrt{p_{0} !}},
$$

due to (4.127) and the following inequality on the interval $\left[T_{0}, T\right]$ :

$$
\left\|\left(u_{n_{0}+1}-u_{n_{0}}\right)(r)\right\|_{\infty} \leq\left\|u_{n_{0}+1}\right\|_{\infty}+\left\|u_{n_{0}}\right\|_{\infty} \leq 2 C_{0}^{*} \leq 2 C_{*} .
$$

Let us now assume that for a given $n$, Inequality (4.131) holds. From Corollary 4.125 and Inequality (4.130), we deduce that for all $t \in\left[T_{0}, \widetilde{T}\right]$

$$
\begin{aligned}
\alpha_{n+1}(t) \leq & C_{*} \int_{t}^{\widetilde{T}} \alpha_{n}(r) d r+C_{*} \sigma_{n+n_{0}}^{*}(t) \sqrt{\widetilde{T}-t} \\
\leq & 2 \frac{C_{*}^{p_{0}+n+1}(\widetilde{T}-t)^{\left(p_{0}+n+2\right) / 2}}{\sqrt{\left(p_{0}+n\right) !}} \frac{2}{p_{0}+n+2} \\
& +\frac{C_{*}^{n+p_{0}+1}(\widetilde{T}-t)^{\frac{n+p_{0}}{2}}}{\sqrt{\left(n+p_{0}+1\right) !}} \sqrt{\widetilde{T}-t} \frac{\sigma_{n+n_{0}}^{*}}{\sigma_{n}^{*}}(t) .
\end{aligned}
$$

But, with (4.129)

$$
\frac{\sigma_{n+n_{0}}^{*}}{\sigma_{n}^{*}}(t)=\frac{C_{*}^{n_{0}}(\widetilde{T}-t)^{\frac{n_{0}}{2}}}{\sqrt{\left(n+p_{0}+2\right) \cdots\left(n+p_{0}+n_{0}\right)}} \leq \frac{C_{*}^{n_{0}}(\widetilde{T}-t)^{\frac{n_{0}}{2}}}{\sqrt{n_{0} !}} \sqrt{\left(p_{0}+1\right) !} \leq 1 .
$$

Thus

$$
\begin{aligned}
& \alpha_{n+1}(t) \leq 2 \frac{C_{*}^{p_{0}+n+1}(\widetilde{T}-t)^{\left(p_{0}+n+1\right) / 2}}{\sqrt{\left(p_{0}+n+1\right) !}} \frac{2 \sqrt{\widetilde{T}-t}}{\sqrt{p_{0}+n+1}} \\
&+\frac{C_{*}^{n+p_{0}+1}(\widetilde{T}-t)^{\frac{n+p_{0}+1}{2}}}{\sqrt{\left(n+p_{0}+1\right) !}}
\end{aligned}
$$

We then conclude with (4.126) that

$$
\alpha_{n+1}(t) \leq 2 \frac{C_{*}^{p_{0}+n+1}(\widetilde{T}-t)^{\left(p_{0}+n+1\right) / 2}}{\sqrt{\left(p_{0}+n+1\right) !}} .
$$


Therefore the series $\sum \alpha_{n}$ is convergent, uniformly on $\left[T_{0}, T\right]$. So the sequence $\left(u_{n}\right)$ uniformly converges on $\left[T_{0}, T\right] \times \mathbb{R}^{d}$.

We now apply Theorem 15 p.80 in [8]. Let us define the following sequence of parabolic operators:

$$
\mathcal{G}_{n}=\partial_{t}+\phi\left(t, x, u_{n}\right) \cdot \nabla+\frac{\sigma_{n}(t)+1}{2} \Delta
$$

We can write:

$$
\mathcal{G}_{n} u_{n+1}=f_{n}+\frac{1}{2} \Delta u_{n+1} .
$$

Let us verify the assumptions of Friedman's Theorem on any bounded domain $\Xi \subset[0, T) \times \mathbb{R}^{d}$ of the form $\Xi=\left[0, T\left[\times[m, M]\right.\right.$. First we know that $\left(u_{n}\right)$ uniformly converges to a function $u$ on $\left[T_{0}, T\right] \times \mathbb{R}^{d}$. Second from Lemma 4.2 we deduce that

$$
\sup _{n \geq 0}\left\|\partial_{t} u_{n}\right\|_{\infty}<\infty
$$

and then

$$
\sup _{n \geq 0}\left(\left\|\partial_{t} u_{n}\right\|_{\infty}+\left\|u_{n}\right\|_{\infty}+\left\|\nabla u_{n}\right\|_{\infty}\right)<\infty
$$

Therefore

$$
\sup _{n \geq 0}\left(\left\|\partial_{t}\left(f_{n}+\frac{1}{2} \Delta u_{n+1}\right)\right\|_{\infty}+\left\|\nabla\left(f_{n}+\frac{1}{2} \Delta u_{n+1}\right)\right\|_{\infty}+\left\|f_{n}+\frac{1}{2} \Delta u_{n+1}\right\|_{\infty}\right)<\infty .
$$

Condition (B) (See Appendix 5.1) i.e. the uniform parabolic condition obviously holds, whereas Condition (A) holds due to (4.2). As a consequence, there exists a subsequence of $\left(u_{n+1}\right)$ such that its two first derivatives also converge uniformly on the domain $\Xi$. Moreover $u$ is continuously twice differentiable and verifies

$$
\left(\mathcal{G}_{u} u\right)(t, x)=\left(\partial_{t} u+\phi(t, x, u) \cdot \nabla u+\frac{1}{2} \Delta u\right)(t, x)=-g(t, x, u(t, x))+\frac{1}{2} \Delta u(t, x), \quad(t, x) \in \Xi .
$$

As a conclusion, using Theorem 10 p.72 in [8], we deduce that $u \in C^{2}\left([0, T) \times \mathbb{R}^{d}\right)$.

Let us prove the uniqueness of the solution. Let $u$ and $v$ be two solutions and write:

$$
\begin{aligned}
\partial_{t} u(t, x)+(\phi(u) \cdot \nabla) u(t, x)+\sigma \Delta u(t, x) & =-g(t, x, u(t, x))+\sigma \Delta u(t, x) \\
\partial_{t} v(t, x)+(\phi(v) \cdot \nabla) v(t, x)+\sigma \Delta v(t, x) & =-g(t, x, v(t, x))+\sigma \Delta v(t, x) .
\end{aligned}
$$

Thus

$$
\begin{aligned}
(u-v)(t, x)= & E \int_{t}^{T}\left\{g\left(s, X_{t, x}^{u}(s), u\left(s, X_{t, x}^{u}(s)\right)\right)-g\left(s, X_{t, x}^{v}(s), u\left(s, X_{t, x}^{u}(s)\right)\right)\right. \\
& +g\left(s, X_{t, x}^{v}(s), u\left(s, X_{t, x}^{u}(s)\right)\right)-g\left(s, X_{t, x}^{v}(s), u\left(s, X_{t, x}^{v}(s)\right)\right) \\
& +g\left(s, X_{t, x}^{v}(s), u\left(s, X_{t, x}^{v}(s)\right)\right)-g\left(s, X_{t, x}^{v}(s), v\left(s, X_{t, x}^{v}(s)\right)\right) \\
& +\sigma\left(\Delta u\left(s, X_{t, x}^{u}(s)\right)-\Delta u\left(s, X_{t, x}^{v}(s)\right)\right) \\
& \left.+\sigma\left(\Delta u\left(s, X_{t, x}^{v}(s)\right)-\Delta v\left(s, X_{t, x}^{v}(s)\right)\right)\right\} d s \\
& +E\left[u_{0}\left(X_{t, x}^{u}(T)\right)-u_{0}\left(X_{t, x}^{v}(T)\right)\right] .
\end{aligned}
$$


We then have

$$
\begin{aligned}
E\left|X_{t, x}^{u}(s)-X_{t, x}^{v}(s)\right| \leq & a_{1} \int_{t}^{T}\|u(r)-v(r)\|_{\infty} d r \\
& +a_{1} \int_{t}^{s} E\left|X_{t, x}^{u}(s)-X_{t, x}^{v}(r)\right| d r
\end{aligned}
$$

Therefore

$$
E\left|X_{t, x}^{u}(s)-X_{t, x}^{v}(s)\right| \leq a_{2} \int_{t}^{T}\|u(r)-v(r)\|_{\infty} d r
$$

As in the previous lemma, we can prove that there exists $C$ such that for all $\sigma \in(0,1)$,

$$
\|(u-v)(t)\|_{\infty} \leq C \int_{t}^{T}\|(u-v)(r)\|_{\infty} d r+\sigma \int_{T_{0}}^{T}\|(\Delta u-\Delta v)(r)\|_{\infty} d r .
$$

We then have by Gronwall's Lemma:

$$
\|(u-v)(t)\|_{\infty} \leq C \sigma \int_{T_{0}}^{T}\|(\Delta u-\Delta v)(r)\|_{\infty} d r,
$$

for all $\sigma>0$. Therefore $u=v$.

\section{Appendix}

5.1. Limit theorem for a sequence of parabolic PDEs. In the following we reformulate, with the change of time $t \longleftrightarrow T-t$, Theorem 15 p. 80 from [8] we need in this paper. For this, we first recall some notation needed for its formulation on a given domain $\Xi \subset[0, T) \times \mathbb{R}^{d}$ of the form $[0, T) \times[m, M]^{d}$. Note that this latter can be chosen arbitrarily and the theorem we shall recall remains true as asserted p. 61 in [8].

We define:

$$
B_{T}:=\{T\} \times[m, M]^{d}, B_{\tau}:=\Xi \cap\{t=\tau\}, S_{\tau}:=S \cap\{t \geq \tau\}, \Xi_{\tau}:=\Xi \cap\{t>\tau\}
$$

where $S=\left[0, T\left[\times\{m, M\}^{d}\right.\right.$ such that $\partial \Xi=\overline{B_{0}} \cup \overline{B_{T}} \cup \bar{S}$. We introduce the following norm of a function $u$ defined on $\Xi$ for a given Holder coefficient $\alpha \in] 0,1[$ :

$$
|u|_{\alpha}^{\Xi}=\sup _{y \in \Xi}|u(y)|+\sup _{P=(t, x), Q=\left(t^{\prime}, x^{\prime}\right) \in \Xi} d_{P, Q}^{\alpha} \frac{|u(P)-u(Q)|}{d^{\alpha}(P, Q)}
$$

where $d(P, Q)$ is the distance on $\mathbb{R}^{d} \times[0, T]$ defined as

$$
d\left[(x, t),\left(x^{\prime}, t^{\prime}\right)\right]=\sqrt{\left|x-x^{\prime}\right|^{2}+\left|t-t^{\prime}\right|}
$$

and for any point $Q=(x, \tau) \in \Xi, P \in \Xi$

$$
d_{Q}:=d\left(Q, B_{0}+S_{\tau}\right), \quad d_{P, Q}:=\min \left\{d_{P}, d_{Q}\right\} .
$$

We also consider

$$
|d v|_{\alpha}^{\Xi}:=\sup _{P \in \Xi} d_{P}|v(P)|+\sup _{P, Q \in \Xi} d_{P, Q}^{1+\alpha} \frac{|v(P)-v(Q)|}{d(P, Q)^{\alpha}} .
$$

(Here the notation $d v$ does not designate a derivative.) Since $\Xi$ is bounded, observe that the quantities $d_{P}, d_{P, Q}$ are uniformly bounded on $P, Q \in \Xi$. Moreover, if the first differential of $v$ is bounded, then $|d v|_{\alpha}^{\Xi}$ is bounded for all $\alpha<1$. 
We consider an operator

$$
L=\sum_{i, j=1}^{n} a_{i, j}(x, t) \frac{\partial^{2}}{\partial x_{i} \partial x_{j}}+\sum_{i=1}^{n} b_{i}(x, t) \frac{\partial}{\partial x_{i}}+\frac{\partial}{\partial t}
$$

defined on $\Xi$ verifying:

(A) The coefficients of $L$ are locally Holder continuous (exponent $\alpha$ ) on $\Xi$, and there is a constant $K_{1}$ such that

$$
\left|a_{i, j}\right|_{\alpha}^{\Xi} \leq K_{1},\left|d b_{i}\right|_{\alpha}^{\Xi} \leq K_{1}
$$

(B) There exists $K_{2}>0$ such that for all $(x, t) \in \Xi$ and $\xi \in \mathbb{R}^{d}$,

$$
\sum_{i, j=1}^{n} a_{i, j}(x, t) \xi_{i} \xi_{j} \geq K_{2}|\xi|^{2}
$$

Theorem 5.1. Let $\left\{L_{m}\right\}_{m}$ be a sequence of parabolic operators satisfying (A), (B) for some constants $K_{1}, K_{2}$ independent of $m$, and let $\left\{f_{m}\right\}_{m}$ be a sequence of functions satisfying $\left|f_{m}\right|_{\alpha}^{\Xi} \leq$ $K_{3}$ where $K_{3}$ is independent of $m$. Suppose that $\left\{u_{m}\right\}_{m}$ is a sequence of functions satisfying

$$
L_{m} u_{m}=f_{m} \text { on } \Xi \text {. }
$$

If $\sup _{y \in \Xi}\left|u_{m}(y)\right| \leq K_{4}$, where $K_{4}$ is independent of $m$, then for any subsequence $\left\{u_{m}^{(1)}\right\}_{m}$ of $\left\{u_{m}\right\}_{m}$ there exists a subsequence of it, say $\left\{u_{m}^{(2)}\right\}_{m}$, such that

$$
u_{m}^{(2)}, \nabla u_{m}^{(2)}, \operatorname{Hess}\left[u_{m}^{(2)}\right], \partial_{t} u_{m}^{(2)}
$$

are uniformly convergent in any subdomain of $\Xi$ to some function $u$ and its corresponding derivatives. Furthermore, $u \in C_{2+\alpha}(\Xi)$. If, in particular, the coefficients of $L_{m}$ converge to the corresponding coefficients of an operator $L$ and $f_{m}$ converges to $f$, pointwise in $\Xi$, then $L u=f$ in $\Xi$.

Acknowledgments: The authors would like to thank many of their colleagues in Stochastic Calculus and PDEs for helpful discussions and references.

\section{REFERENCES}

[1] Ambrosio, L.; Figalli, A. On flows associated to Sobolev vector fields in Wiener spaces: an approach à la DiPerna-Lions. J. Funct. Anal. 256 (2009), no. 1, 179-214.

[2] Belopol'skaya, Ya. A probability approach to the method of vanishing viscosity. Applications of mathematical analysis. J. Math. Sci. (N.Y.) 120 (2004), no. 2, 1051-1079.

[3] Belopol'skaya, Ya.; Woyczynski, W. A. Generalized solutions of nonlinear parabolic equations and diffusion processes. Acta Appl. Math. 96 (2007), no. 1-3, 55-69.

[4] Bianchini, S., Bressan, A. Vanishing viscosity solutions of nonlinear hyperbolic systems. Ann. of Math. (2) 161 (2005), no. 1, 223-342.

[5] Busnello B., A probabilistic approach to the two-dimensional Navier-Stokes equations. Ann. Probab. 27 (1999), no. 4, 1750-1780.

[6] Delarue, F. On the existence and uniqueness of solutions to FBSDEs in a non-degenerate case. Stochastic Process. Appl. 99 (2002), no. 2, 209-286.

[7] Evans, L. C. Partial differential equations. Graduate Studies in Mathematics, 19. American Mathematical Society, Providence, RI, 1998.

[8] Friedman, A. Partial differential equations of parabolic type. Prentice-Hall, Inc., Englewood Cliffs, N.J. 1964. 347 pp. 
[9] Friedman, A. Stochastic differential equations and applications. Vol. 1. Probability and Mathematical Statistics, Vol. 28. Academic Press [Harcourt Brace Jovanovich, Publishers], New York-London, 1975. $231 \mathrm{pp}$.

[10] Henry, D. Geometric theory of semilinear parabolic equations. Lecture Notes in Mathematics, 840. Springer-Verlag, Berlin-New York, 1981.

[11] Kunita, H. Stochastic flows and stochastic differential equations. Cambridge Studies in Advanced Mathematics, 24. Cambridge University Press, Cambridge, 1990.

[12] Ladyženskaja, O. A.; Solonnikov, V. A.; Ural'ceva, N. N. Linear and quasilinear equations of parabolic type. (Russian) Translated from the Russian by S. Smith. Translations of Mathematical Monographs, Vol. 23 American Mathematical Society, Providence, R.I. 1967 xi+648 pp.

[13] Ma, J.; Yong, J. Forward-backward stochastic differential equations and their applications. Lecture Notes in Mathematics, 1702. Springer-Verlag, Berlin, 1999. xiv+270 pp.

[14] Ma, J.; Zhang, J. Representation theorems for backward stochastic differential equations. Ann. Appl. Probab. 12 (2002), no. 4, 1390-1418.

[15] Majda, A. Compressible fluid flow and systems of conservation laws in several space variables. Applied Mathematical Sciences, 53. Springer-Verlag, New York, 1984.

[16] Métivier, G. The Mathematics of Nonlinear Optics. http://www.math.ubordeaux1.fr/ metivier/cours.html

[17] Pardoux, É. Backward stochastic differential equations and viscosity solutions of systems of semilinear parabolic and elliptic PDEs of second order. Stochastic analysis and related topics, VI (Geilo, 1996), 79-127, Progr. Probab., 42, Birkhuser Boston, Boston, MA, 1998.

[18] Taylor, M. E. Partial differential equations. III. Nonlinear equations. Corrected reprint of the 1996 original. Applied Mathematical Sciences, 117. Springer-Verlag, New York, 1997.

[19] Wiegner, M. Global solutions to a class of strongly coupled parabolic systems. Math. Ann. 292 (1992), no. $4,711-727$.

LATP, UmR 6632, Université de Provence, Technopôle Chteau-Gombert, 39 rue Joliot Curie, 13453 Marseille Cedex 13, France

E-mail address: darses@cmi.univ-mrs.fr

Cérémade, Umr 7534, Université Paris Dauphine, Place du Marchal De lattre De TasSigny, 75775 Paris CedeX 16, France

E-mail address: emmanuel.denis@ceremade.dauphine.fr 